\title{
Dynamic analysis of a complex system under preemptive repeat repair discipline
}

\author{
Ehmet Kasim" and Geni Gupur ${ }^{1}$
}

"Correspondence:

ehmetkasim@163.com

${ }^{1}$ College of Mathematics and

Systems Science, Xinjiang

University, Urumqi, P.R. China

\section{Springer}

\begin{abstract}
This paper considers a two-unit complex system, in which one of the components has priority with preemptive repeat repair disciplines, described by partial differential equations with integral boundary conditions. First, we prove that the system has a unique nonnegative time-dependent solution by using the strong continuous semigroup theory of linear operators. Then, we obtain the exponential convergence of the time-dependent solution to its steady-state solution by means of the spectral properties of the corresponding operator. We also provide the asymptotic behavior of some time-dependent reliability indices, a numerical illustration showing the effects of various parameters on the system, and the validity of the theoretical analysis.
\end{abstract}

Keywords: Two-unit system with priority; $C_{0}$-semigroup; Well-posedness; Asymptotic behavior; Reliability indices

\section{Introduction}

In many real-life situations, system reliability plays a very important role. With an increasing complexity of industrial systems, the reliability-related problems are quite challenging due to the diversity of factors that can lead to failures in industrial systems. Throughout the history of reliability theory, a large number of problems were solved by using reliability models. These models can be developed by means of different methods. Among them we can highlight the supplementary variable technique, introduced in 1955 by Cox [1], due to the fact that this technique outperforms imbedded Markov chains in the steady-state case. The supplementary variable technique was firstly applied in Gaver [2]; subsequently, other authors, such as Linton [3], Goel et al. [4], Gupta and Sharma [5], Shi and Li [6], Chung [7], Yuan [8], Dhillon and Cheng [9], Ram et al. [10], Zhang and Wang [11], Ke et al. [12], followed this line of research.

The supplementary variable technique allows to transform a continuous-time nonMarkovian process into a Markovian one by including one or more supplementary variables. However, this technique leads to mathematical models described by partial differential equations with integral boundary conditions, and therefore, there are significant difficulties to obtain exact solutions. Due to this fact, some papers related to reliability models assume that time-dependent solutions converge to their steady-state solutions, but they do not answer whether this assumption holds. Hence, we need a study of the existence of

(c) The Author(s) 2020. This article is licensed under a Creative Commons Attribution 4.0 International License, which permits use sharing, adaptation, distribution and reproduction in any medium or format, as long as you give appropriate credit to the original author(s) and the source, provide a link to the Creative Commons licence, and indicate if changes were made. The images or other third party material in this article are included in the article's Creative Commons licence, unless indicated otherwise in a credit line to the material. If material is not included in the article's Creative Commons licence and your intended use is not permitted by statutory regulation or exceeds the permitted use, you will need to obtain permission directly from the copyright holder. To view a copy of this licence, visit http://creativecommons.org/licenses/by/4.0/. 
time-dependent solutions of reliability models, of time-dependent reliability indices, and of their asymptotic behavior. In 2001, Gupur [13] firstly did dynamic analysis for reliability models, which was established by the supplementary variable technique, by means of the $C_{0}$-semigroup theory. After that, he and his coauthors studied several reliability models. For example, Gupur and Li [14] considered the system which consists of a reliable machine, an unreliable machine, and a storage buffer with infinite capacity and obtained the existence of a unique positive time-dependent solution of the system. By using Greiner's idea, Gupur and Wang [15] studied spectra of the operator on the imaginary axis and proved that the time-dependent solution of the system strongly converges to its steadystate solution. In [16], Gupur described the point spectrum of the operator which corresponds to the system. In 2003, Gupur [17] investigated the well-posedness of a repairable, standby, human, and machine system. Gupur [18] studied asymptotic behavior of the system and obtained that the time-dependent solution of the system converges strongly to its steady-state solution. Aili and Gupur [19] did further analysis and concluded that the time-dependent solution of the system exponentially converges to its steady-state solution. Ehmet and Gupur [20] examined the k-out-of-N:G redundant system with repair and multiple critical and noncritical errors and proved that the corresponding $C_{0}$-semigroup is quasi-compact and converges exponentially to a projection operator. Further, Ablet and Gupur [21] gave the explicit expression of the above projection operator and deduced that the time-dependent solution of the system exponentially converges to its steady-state solution when the repair rates are constant. In combination with the above-mentioned results, these research ideas and methods were introduced in detail in Gupur's book [22]. During the past decade, inspired by the new research methods, many authors have done remarkable contributions on the dynamic analysis of different reliability models, see [23-26] and the references therein.

The maintenance of system equipment is a key factor for the smooth operation of an industrial system that usually requires the design of various methods and means in order to improve the availability of the system. One of these methods is to assign priority according to the nature of components of the system. Govil [27] considered the system composed of two types (denoted hereafter as Type I and II) of components, in which Type I component has the priority with preemptive repeat repair disciplines. The model proposed in that paper was based on the supplementary variable technique and gave the Laplace transform of the probability generating function which was defined by the time-dependent solution. The effect of different repair priorities on the pointwise availability of the system was investigated for a special case with numerical examples. To the best of our knowledge, there are no further results related to this work. In this paper, we do dynamic analysis for the Govil [27] model.

First of all, we convert the corresponding system into an abstract Cauchy problem in a Banach space and prove the well-posedness of the system by showing that the underlying operator generates contraction $C_{0}$-semigroup, i.e., the system has a unique positive time-dependent solution which satisfies the probability condition. Then, we investigate the asymptotic behavior of the time-dependent solution and obtain that the $C_{0^{-}}$ semigroup generated by the underlying operator is a quasi-compact operator. We also prove that 0 is an eigenvalue of the operator with algebraic multiplicity one, and therefore, the $C_{0}$-semigroup converges exponentially to a projection operator. Hence, we deduce that the time-dependent solution of the system converges strongly to its steady-state so- 
lution. Next, we use an idea taken from Gupur $[19,28]$ to show that the essential growth bound of the $C_{0}$-semigroup is less than a negative number, thus we deduce that 0 is an isolated eigenvalue of the operator and pole of order 1 . In addition, by using the residue theorem, we determine the expression of the projection operator and conclude that the time-dependent solution of the system converges exponentially to its steady-state solution. Finally, we discuss the asymptotic behavior of the time-dependent reliability indices, such as time-dependent availability, failure frequency, renewal frequency, and reliability of the system, and illustrate, with numerical examples, the effect of changes in the system parameters on those indices in a particular case.

\section{Mathematical model of the system}

In this section we firstly give a detailed description of the system and then present a model for it based on the supplementary variables technique. Next, we convert the model into an abstract Cauchy problem.

\subsection{Assumptions and description of the model}

The assumptions of our mathematical model are the following.

(1) In the system there are two types of components denoted as Type I and Type II.

(2) Component of Type I is always given preference over Type II component.

(3) Non-failed component cannot fail when the system fails.

(4) The failure behavior of Type I component is unaffected by the failure of Type II component, if any.

(5) On failure of Type II component, the system works in a degraded state, but does not fail.

(6) The system can fail completely due to failure of Type I component.

(7) When the Type II component is failed, its repair is interrupted (preempted) when Type I component fails (and the system thus fails). When the Type I component is finally repaired, repair of the Type II component begins all over, as if the Type II component has just failed (the previous repair time was for naught). This implies that the repair of the Type II component-when rebegun-is considered as the repair of the freshly failed component.

(8) All the failures follow the exponential distribution, $\lambda, \lambda^{\prime}$ denote the failure rates of components of Type I and Type II, respectively.

(9) All the repairs follow the general distribution. $\eta(x)$ represents the repair rate of a component of Type I and satisfies $\eta(x) \geq 0, \int_{0}^{\infty} \eta(x) d x=\infty . \varphi(x)$ represents the repair rate of a component of Type II and satisfies $\varphi(x) \geq 0, \int_{0}^{\infty} \varphi(x) d x=\infty$.

(10) All the above random variables are independent. The switching device is perfect.

Let $S(t)$ be a random variable representing the state of the system at time t. Since the distributions of the repair time are assumed as general distributions, $S(t)$ is not Markovian in continuous time. However, after introducing supplementary variables $Y(t)$, the process $\{S(t), Y(t) \mid t>0\}$ becomes Markovian, where $Y(t)$ is the elapsed repair time of the failed component. According to the previous assumptions, this system has the following possible states:

$\mathbf{S}(\mathbf{t})=\mathbf{0}:$ All components are good, the system is operating.

$\mathbf{S}(\mathbf{t})=\mathbf{1}$ : The component of Type II is failed, the system is degraded.

$\mathbf{S}(\mathbf{t})=\mathbf{2}$ : The component of Type I is failed, the system is failed. 
Figure 1 State transition diagram of the system

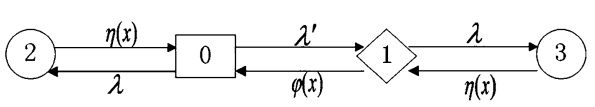

cood State

Degraded State

Failed State

$\mathbf{S}(\mathbf{t})=3$ : The component of Type I is failed and the component of Type II is waiting repair, the system is failed.

The transitions between states of the system are shown in Fig. 1.

Before presenting the model, we need the following definitions:

$P_{0}(t)$ is the probability that at time $t$ the system is operating in normal efficiency.

$P_{1}(x, t) \Delta x$ is the probability that at time $t$ the system is in the degraded state due to the failure of a component of Type II, and the elapsed repair time lies in the interval $(x, x+\Delta x)$.

$P_{2}(x, t) \Delta x$ is the probability that at time the system is in the failed state due to the failure of a component of Type I, and the elapsed repair time lies in the interval $(x, x+\Delta x)$.

$P_{3}(x, t) \Delta x$ is the probability that at time $t$ the system is still failed due to the failure of a component of Type I; and the elapsed repair time lies in the interval $(x, x+\Delta x)$ at the instant when it preempted in the repair facility and Type II, which had been in service, is waiting repair.

By the supplementary variable technique, the above system can be described by the following system of partial differential equations (see Govil [27]):

$$
\begin{aligned}
& \frac{d P_{0}(t)}{d t}=-\left(\lambda+\lambda^{\prime}\right) P_{0}(t)+\int_{0}^{\infty} P_{1}(x, t) \varphi(x) d x+\int_{0}^{\infty} P_{2}(x, t) \eta(x) d x, \\
& \frac{\partial P_{1}(x, t)}{\partial t}+\frac{\partial P_{1}(x, t)}{\partial x}=-(\lambda+\varphi(x)) P_{1}(x, t), \\
& \frac{\partial P_{j}(x, t)}{\partial t}+\frac{\partial P_{j}(x, t)}{\partial x}=-\eta(x) P_{j}(x, t), \quad j=2,3,
\end{aligned}
$$

with the integral boundary conditions:

$$
\begin{aligned}
& P_{1}(0, t)=\lambda^{\prime} P_{0}(t)+\int_{0}^{\infty} P_{3}(x, t) \eta(x) d x, \\
& P_{2}(0, t)=\lambda P_{0}(t), \\
& P_{3}(0, t)=\lambda \int_{0}^{\infty} P_{1}(x, t) d x,
\end{aligned}
$$

and the initial conditions:

$$
P_{0}(0)=1, \quad P_{i}(x, 0)=0, \quad i=1,2,3,
$$

where $(x, t) \in[0, \infty) \times[0, \infty)$.

\subsection{Reset the model}

In this subsection, we convert the above model into an abstract Cauchy problem on a suitable Banach space by introducing underlying operators and their domains. For simplicity, 
we introduce the following notation:

$$
\Upsilon=\left(\begin{array}{cccc}
e^{-x} & 0 & 0 & 0 \\
\lambda^{\prime} e^{-x} & 0 & 0 & \eta(x) \\
\lambda e^{-x} & 0 & 0 & 0 \\
0 & \lambda & 0 & 0
\end{array}\right)
$$

We take a state space as follows:

$$
\mathcal{X}=\left\{\begin{array}{l}
\mathbf{P} \mid \begin{array}{l}
\mathbf{P} \in \mathbb{R} \times L^{1}[0, \infty) \times L^{1}[0, \infty) \times L^{1}[0, \infty) \\
\|\mathbf{P}\|=\left|P_{0}\right|+\sum_{i=1}^{3}\left\|P_{i}\right\|_{L^{1}[0, \infty)}<\infty
\end{array}
\end{array}\right\}
$$

It is obvious that $\mathcal{X}$ is a Banach space. Now we define operators and their domain as follows:

$$
\begin{aligned}
& D(\mathbb{A})=\left\{\begin{array}{ll}
\mathbf{P} \in \mathcal{X} \mid \begin{array}{l}
\frac{d P_{i}(x)}{d x} \in L^{1}[0, \infty), P_{i}(x) \text { are absolutely } \\
\text { continuous and } \mathbf{P}(0)=\int_{0}^{\infty} \Upsilon \mathbf{P}(x) d x
\end{array}
\end{array}\right\} \\
& \mathbb{A}\left(\begin{array}{c}
P_{0} \\
P_{1}(x) \\
P_{2}(x) \\
P_{3}(x)
\end{array}\right)=\left(\begin{array}{cccc}
\left(\lambda+\lambda^{\prime}\right) & 0 & 0 & 0 \\
0 & -\frac{d}{d x}-(\lambda+\varphi(x)) & 0 & \\
0 & 0 & -\frac{d}{d x}-\eta(x) & 0 \\
0 & 0 & 0 & -\frac{d}{d x}-\eta(x)
\end{array}\right)\left(\begin{array}{c}
P_{0} \\
P_{1}(x) \\
P_{2}(x) \\
P_{3}(x)
\end{array}\right), \\
& \mathbb{E}\left(\begin{array}{c}
P_{0} \\
P_{1}(x) \\
P_{2}(x) \\
P_{3}(x)
\end{array}\right)=\left(\begin{array}{c}
\int_{0}^{\infty} P_{1}(x) \varphi(x) d x+\int_{0}^{\infty} P_{2}(x) \eta(x) d x \\
0 \\
0 \\
0
\end{array}\right), \quad D(\mathbb{E})=\mathcal{X} .
\end{aligned}
$$

Then the above system of equations (2.1)-(2.3) can be written as the following abstract Cauchy problem in the Banach space $\mathcal{X}$ :

$$
\left\{\begin{array}{l}
\frac{d \mathbf{P}(t)}{d t}=(\mathbb{A}+\mathbb{E}) \mathbf{P}(t), \quad t \in(0, \infty) \\
\mathbf{P}(0)=(1,0,0,0)^{T}
\end{array}\right.
$$

\section{Well-posedness of system (2.4)}

In this section, we first prove that $\mathbb{A}+\mathbb{E}$ generates a positive contraction $C_{0}$-semigroup $\mathbb{T}(t)$ on $\mathcal{X}$, then we determine the dual space of $\mathcal{X}$, and prove that $\mathbb{A}+\mathbb{E}$ is a conservative operator, which together with the Fattorini theorem [29, P. 155] allows us to deduce that $\mathbb{T}(t)$ is isometric. Finally, from these results we present the well-posedness of system (2.4).

Theorem 3.1 If $\varphi(x)$ and $\eta(x)$ satisfy $\bar{\varphi}=\sup _{x \in[0, \infty)} \varphi(x)<\infty$ and $\bar{\eta}=\sup _{x \in[0, \infty)} \eta(x)<\infty$, then $\mathbb{A}+\mathbb{E}$ generates a positive contraction $C_{0}$-semigroup $\mathbb{T}(t)$.

A detailed proof of Theorem 3.1 can be found in the Appendix. It is not difficult to verify that $\mathcal{X}^{*}$, the dual space of $\mathcal{X}$, is as follows:

$$
\mathcal{X}^{*}=\left\{\begin{array}{l}
\mathbf{Q}^{*} \mid \begin{array}{l}
\mathbf{Q}^{*}=\left(Q_{0}^{*}, Q_{1}^{*}(x), Q_{2}^{*}(x), Q_{3}^{*}(x)\right)^{T} \\
\left\|\mathbf{Q}^{*} \mid\right\|=\sup \left\{\left|Q_{0}^{*}\right|, \sup _{1 \leq i \leq 3}\left\|Q_{i}^{*}\right\|_{L^{\infty}[0, \infty)}\right\}<\infty
\end{array}
\end{array}\right\}
$$


Clearly, $\mathcal{X}^{*}$ is a Banach space. In $\mathcal{X}$ we define the subset

$$
Y=\left\{\begin{array}{l|l}
\mathbf{P} \in \mathcal{X} & \begin{array}{l}
\mathbf{P}(x)=\left(P_{0}, P_{1}(x), P_{2}(x), P_{3}(x)\right), \\
P_{0} \geq 0, P_{i}(x) \geq 0, i=1,2,3, \forall x \in[0, \infty)
\end{array}
\end{array}\right\} .
$$

Then Theorem 3.1 ensures that $T(t) Y \subset Y$. For $\mathbf{P} \in D(\mathbb{A}) \cap Y$, we choose $q^{*}(x)=$ $\|\mathbf{P}\|(1,1,1,1)^{T}$, then $q^{*} \in \mathcal{X}^{*}$ and

$$
\begin{aligned}
\left\langle(\mathbb{A}+\mathbb{E}) \mathbf{P}, q^{*}\right\rangle= & \|\mathbf{P}\|\left\{-\left(\lambda+\lambda^{\prime}\right) p_{0}+\int_{0}^{\infty} \varphi(x) p_{1}(x) d x+\int_{0}^{\infty} \eta(x) p_{2}(x) d x\right\} \\
& +\|\mathbf{P}\| \int_{0}^{\infty}\left\{-\frac{d p_{1}(x)}{d x}-\lambda p_{1}(x)-\varphi(x) p_{1}(x)\right\} d x \\
& +\|\mathbf{P}\| \sum_{j=2}^{3} \int_{0}^{\infty}\left\{-\frac{d p_{j}(x)}{d x}-\eta(x) p_{j}(x)\right\} d x \\
= & \|\mathbf{P}\|\left\{-\left(\lambda+\lambda^{\prime}\right) p_{0}+\int_{0}^{\infty} \varphi(x) p_{1}(x) d x+\int_{0}^{\infty} \eta(x) p_{2}(x) d x\right\} \\
& +\|\mathbf{P}\|\left\{\lambda^{\prime} p_{0}+\int_{0}^{\infty} \eta(x) p_{3}(x) d x-\lambda \int_{0}^{\infty} p_{1}(x) d x-\int_{0}^{\infty} \varphi(x) p_{1}(x) d x\right\} \\
& +\|\mathbf{P}\|\left\{\lambda p_{0}+\int_{0}^{\infty} \lambda p_{1}(x) d x-\int_{0}^{\infty} \eta(x) p_{2}(x) d x-\int_{0}^{\infty} \eta(x) p_{3}(x) d x\right\} \\
= & 0, \quad
\end{aligned}
$$

which implies that $\mathbb{A}+\mathbb{E}$ is conservative with respect to the set

$$
\Theta(\mathbf{P})=\left\{q^{*} \in \mathcal{X}^{*} \mid\left\langle\mathbf{P}, q^{*}\right\rangle=\|P\|^{2}=\left\|q^{*}\right\|^{2}\right\} .
$$

Since the initial value $\mathbf{P}(0) \in D\left(\mathbb{A}^{2}\right) \cap Y$, by using the Fattorini theorem [29, P. 155], we deduce the following result.

Theorem 3.2 $\mathbb{T}(t)$ is isometric for the initial value of system (2.4), that is,

$$
\|\mathbb{T}(t) \mathbf{P}(0)\|=\|\mathbf{P}(0)\|, \quad \forall t \in[0, \infty)
$$

From Theorem 3.1 and Theorem 3.2 we obtain the well-posedness of system (2.4).

Theorem 3.3 If $\varphi(x)$ and $\eta(x)$ satisfy $\bar{\varphi}=\sup _{x \in[0, \infty)} \varphi(x)<\infty$ and $\bar{\eta}=\sup _{x \in[0, \infty)} \eta(x)<\infty$, then system (2.4) has a unique positive time-dependent solution $\mathbf{P}(x, t)$ satisfying

$$
\|\mathbf{P}(\cdot, t)\|=1, \quad \forall t \in[0, \infty)
$$

Proof Since the initial value $\mathbf{P}(0) \in D\left(\mathbb{A}^{2}\right) \cap Y$, from Theorem 3.1 and [22, Theorem 1.81], we know that system (1.4) has a unique positive time-dependent solution $\mathbf{P}(x, t)$ which can be expressed as

$$
\mathbf{P}(x, t)=\mathbb{T}(t) \mathbf{P}(0), \quad t \in[0, \infty) .
$$


From (3.1) and (3.2) we derive

$$
\|\mathbf{P}(\cdot, t)\|=\|\mathbb{T}(t) \mathbf{P}(0)\|=\|\mathbf{P}(0)\|=1, \quad \forall t \in[0, \infty) .
$$

Expression (3.3) reflects the physical background of $\mathbf{P}(x, t)$.

\section{Asymptotic behavior of the time-dependent solution of system (2.4)}

A trivial verification, based on the proof of Theorem 3.1 in the Appendix, shows that the operator $\mathbb{A}$ also generates a positive contraction $C_{0}$-semigroup $\mathbb{S}(t)$. In the following, we first prove that $\mathbb{S}(t)$ is a quasi-compact operator and then, by using the compactness of $\mathbb{E}$, we obtain that $\mathbb{T}(t)$ is a quasi-compact operator. Next we prove that 0 is an eigenvalue of $\mathbb{A}+\mathbb{E}$ and $(\mathbb{A}+\mathbb{E})^{*}$ with geometric multiplicity 1 , thus by using [22, Theorem 1.90 ] we deduce that $\mathbb{T}(t)$ converges exponentially to a projection operator $\mathbb{P r}$, and thereafter determine its explicit expression. Lastly, combining these results, we obtain that the timedependent solution of system (2.4) converges exponentially to its steady-state solution.

Lemma 4.1 If $\mathbf{P}(x, t)=(\mathbb{S}(t) \vartheta)(x)$ is a solution of the following system

$$
\begin{aligned}
& \frac{d \mathbf{P}}{d t}=\mathbb{A} \mathbf{P}(t), \quad t \in[0, \infty), \\
& \mathbf{P}(0)=\vartheta \in D(\mathbb{A}),
\end{aligned}
$$

then

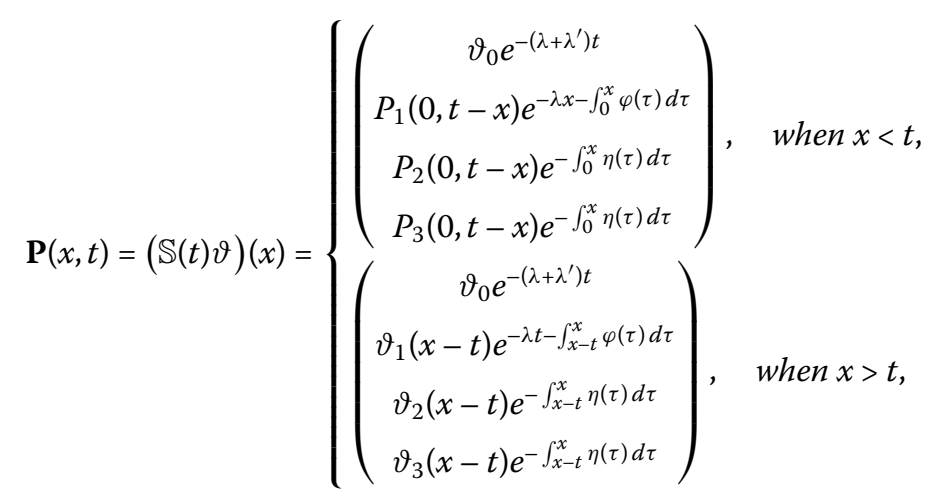

where $P_{i}(0, t-x)(i=1,2,3)$ is given by boundary conditions (2.2).

Proof Since $\mathbf{P}(x, t)=(\mathbb{S}(t) \vartheta)(x)$ is a solution of system $(4.1), \mathbf{P}(x, t)$ satisfies

$$
\begin{aligned}
& \frac{d P_{0}(t)}{d t}=-\left(\lambda+\lambda^{\prime}\right) P_{0}(t), \\
& \frac{\partial P_{1}(x, t)}{\partial t}+\frac{\partial P_{1}(x, t)}{\partial x}=-(\lambda+\varphi(x)) P_{1}(x, t), \\
& \frac{\partial P_{j}(x, t)}{\partial t}+\frac{\partial P_{j}(x, t)}{\partial x}=-\eta(x) P_{j}(x, t), \quad j=2,3, \\
& P_{1}(0)=\lambda^{\prime} P_{0}(t)+\int_{0}^{\infty} P_{3}(x, t) \eta(x) d x,
\end{aligned}
$$




$$
\begin{aligned}
& P_{2}(0, t)=\lambda P_{0}(t) \\
& P_{3}(0, t)=\lambda \int_{0}^{\infty} P_{1}(x, t) d x \\
& P_{0}(0)=\vartheta_{0}, P_{i}(x, 0)=\vartheta_{i}(x), \quad i=1,2,3 .
\end{aligned}
$$

Take $\omega=x-t$ and $Q_{i}(t)=P_{i}(\omega+t, t), i=1,2,3$, then (4.3)-(4.4) gives

$$
\begin{aligned}
& \frac{d Q_{1}(t)}{d t}=-(\lambda+\varphi(\omega+t)) Q_{1}(t), \\
& \frac{d Q_{j}(t)}{d t}=-\eta(\omega+t) Q_{j}(t), \quad j=2,3 .
\end{aligned}
$$

If $\omega<0$ (i.e., $x<t$ ), then by integrating (4.9)-(4.10) from $-\omega$ to $t$ separately and using $Q_{i}(-\omega)=P_{i}(0,-\omega)=P_{i}(0, t-x), i=1,2,3$, we have

$$
\begin{aligned}
P_{1}(x, t) & =Q_{1}(t)=Q_{1}(-\omega) e^{-\lambda(\omega+t)-\int_{-\omega}^{t} \varphi(\omega+\tau) d \tau} \\
& \stackrel{x=\omega+\tau}{=} P_{1}(0, t-x) e^{-\lambda x-\int_{0}^{x} \varphi(\tau) d \tau}, \\
P_{j}(x, t) & =Q_{j}(t)=Q_{j}(-\omega) e^{-\int_{-\omega}^{t} \eta(\omega+\tau) d \tau} \\
& =P_{j}(0, t-x) e^{-\int_{0}^{x} \eta(\tau) d \tau}, \quad j=2,3 .
\end{aligned}
$$

Combining (4.2) with (4.8) we obtain

$$
P_{0}(t)=\vartheta_{0} e^{-\left(\lambda+\lambda^{\prime}\right) t}
$$

If $\omega>0$ (i.e., $x>t$ ), then integrating (4.9)-(4.10) from 0 to $t$ and using the relations $Q_{i}(0)=$ $P_{i}(\omega, 0)=\vartheta_{i}(t-x), i=1,2,3$, and a similar argument to (4.11)-(4.12) we deduce

$$
\begin{aligned}
P_{1}(x, t) & =Q_{1}(t)=Q_{1}(0) e^{-\lambda t-\int_{0}^{t} \varphi(\omega+\tau) d \tau} \\
& \stackrel{\sigma=\omega+\tau}{=} \vartheta_{1}(x-t) e^{-\lambda t-\int_{\omega}^{\omega+t} \varphi(\sigma) d \sigma} \\
& =\vartheta_{1}(x-t) e^{-\lambda t-\int_{x-t}^{x} \varphi(\tau) d \tau}, \\
P_{j}(x, t) & =Q_{j}(t)=Q_{j}(0) e^{-\int_{0}^{t} \eta(\omega+\tau) d \tau} \\
& \stackrel{\sigma=\omega+\tau}{=} \vartheta_{j}(x-t) e^{-\int_{\omega}^{\omega+t} \eta(\sigma) d \sigma} \\
& =\vartheta_{j}(x-t) e^{-\int_{x-t}^{x} \eta(\tau) d \tau}, \quad j=2,3 .
\end{aligned}
$$

(4.11)-(4.15) complete the proof.

Now, we will prove that $\mathbb{S}(t)$ is a quasi-compact operator on $\mathcal{X}$. To this end, we define the following two operators for $\mathbf{P} \in \mathcal{X}$ :

$$
(\mathbb{V}(t) \mathbf{P})(x)= \begin{cases}0 & x \in[0, t), \\ (\mathbb{S}(t) \mathbf{P})(x), & x \in[t, \infty),\end{cases}
$$




$$
(\mathbb{W}(t) \mathbf{P})(x)= \begin{cases}(\mathbb{S}(t) \mathbf{P})(x), & x \in[0, t), \\ 0, & x \in[t, \infty)\end{cases}
$$

Obviously,

$$
(\mathbb{S}(t) \mathbf{P})(x)=(\mathbb{V}(t) \mathbf{P})(x)+(\mathbb{W}(t) \mathbf{P})(x), \quad \forall \mathbf{P} \in \mathcal{X}
$$

From [22, Th. 1.35], it is not difficult to obtain the following result.

Lemma 4.2 A bounded subset $F \subset \mathcal{X}$ is relatively compact if and only if the following two conditions hold simultaneously:

$$
\begin{aligned}
& \text { (1) } \lim _{h \rightarrow 0} \sum_{n=1}^{3} \int_{0}^{\infty}\left|f_{n}(x+h)-f_{n}(x)\right| d x=0, \quad \text { uniformly for } f=\left(f_{0}, f_{1}, f_{2}, f_{3}\right) \in F ; \\
& \text { (2) } \lim _{h \rightarrow \infty} \sum_{n=1}^{3} \int_{h}^{\infty}\left|f_{n}(x)\right| d x=0, \quad \text { uniformly for } f=\left(f_{0}, f_{1}, f_{2}, f_{3}\right) \in F .
\end{aligned}
$$

Theorem 4.1 Assume that $\varphi(x)$ and $\eta(x)$ are Lipschitz continuous and that there exist positive constants $\underline{\varphi}, \bar{\varphi}$ and $\underline{\eta}, \bar{\eta}$ such that

$$
0<\underline{\varphi} \leq \varphi(x) \leq \bar{\varphi}<\infty, \quad 0<\underline{\eta} \leq \eta(x) \leq \bar{\eta}<\infty,
$$

then $\mathbb{W}(t)$ is a compact operator on $\mathcal{X}$.

Proof It is sufficient to prove condition (1) in Lemma 4.2. For bounded $\vartheta \in \mathcal{X}$, we set $\mathbf{P}(x, t)=(\mathbb{S}(t) \vartheta)(x), x \in[0, t)$, then $\mathbf{P}(x, t)$ is a generalized solution of system (4.1). Hence, according to Lemma 4.1 we have, for $x \in[0, t), h \in(0, t], x+h \in[0, t)$,

$$
\begin{aligned}
& \sum_{i=1}^{3} \int_{0}^{t}\left|P_{i}(x+h, t)-P_{i}(x, t)\right| d x \\
&=\int_{0}^{t} \mid P_{1}(0, t-x-h) e^{-\lambda(x+h)-\int_{0}^{x+h} \varphi(\tau) d \tau}-P_{1}(0, t-x-h) e^{-\lambda x-\int_{0}^{x} \varphi(\tau) d \tau} \\
& \quad+P_{1}(0, t-x-h) e^{-\lambda x-\int_{0}^{x} \varphi(\tau) d \tau}-P_{1}(0, t-x) e^{-\lambda x-\int_{0}^{x} \varphi(\tau) d \tau} \mid d x \\
& \quad+\int_{0}^{t} \mid P_{2}(0, t-x-h) e^{-\int_{0}^{x+h} \eta(\tau) d \tau}-P_{2}(0, t-x-h) e^{-\int_{0}^{x} \eta(\tau) d \tau} \\
& \quad+P_{2}(0, t-x-h) e^{-\int_{0}^{x} \eta(\tau) d \tau}-P_{2}(0, t-x) e^{-\int_{0}^{x} \eta(\tau) d \tau} \mid d x \\
& \quad+\int_{0}^{t} \mid P_{3}(0, t-x-h) e^{-\int_{0}^{x+h} \eta(\tau) d \tau}-P_{3}(0, t-x-h) e^{-\int_{0}^{x} \eta(\tau) d \tau} \\
& \quad+P_{3}(0, t-x-h) e^{-\int_{0}^{x} \eta(\tau) d \tau}-P_{3}(0, t-x) e^{-\int_{0}^{x} \eta(\tau) d \tau} \mid d x \\
& \leq \int_{0}^{t}\left|P_{1}(0, t-x-h)\right| \mid e^{-\lambda(x+h)-\int_{0}^{x+h} \varphi(\tau) d \tau}-e^{-\lambda x-\int_{0}^{x} \varphi(\tau) d \tau \mid d x} \\
&+\int_{0}^{t}\left|P_{1}(0, t-x-h)-P_{1}(0, t-x)\right|\left|e^{-\lambda x-\int_{0}^{x} \varphi(\tau) d \tau}\right| d x
\end{aligned}
$$




$$
\begin{aligned}
& +\int_{0}^{t}\left|P_{2}(0, t-x-h)\right|\left|e^{-\int_{0}^{x+h} \eta(\tau) d \tau}-e^{-\int_{0}^{x} \eta(\tau) d \tau}\right| d x \\
& +\int_{0}^{t}\left|P_{2}(0, t-x-h)-P_{2}(0, t-x)\right|\left|e^{-\int_{0}^{x} \eta(\tau) d \tau}\right| d x \\
& +\int_{0}^{t}\left|P_{3}(0, t-x-h)\right|\left|e^{-\int_{0}^{x+h} \eta(\tau) d \tau}-e^{-\int_{0}^{x} \eta(\tau) d \tau}\right| d x \\
& +\int_{0}^{t}\left|P_{3}(0, t-x-h)-P_{3}(0, t-x)\right|\left|e^{-\int_{0}^{x} \eta(\tau) d \tau}\right| d x
\end{aligned}
$$

In the following we estimate each term in (4.16). By using the boundary conditions and properties of the semigroup, we have

$$
\begin{aligned}
& \left|P_{1}(0, t-x-h)\right| \leq\left|\lambda^{\prime} P_{0}(t-x-h)\right|+\bar{\eta} \int_{0}^{\infty}\left|P_{3}(s, t-x-h)\right| d s \\
& \leq \max \left\{\lambda^{\prime}, \bar{\eta}\right\}\left\{\left|P_{0}(t-x-h)\right|+\int_{0}^{\infty}\left|P_{3}(s, t-x-h)\right| d s\right\} \\
& =\max \left\{\lambda^{\prime}, \bar{\eta}\right\}\|P(\cdot, t-x-h)\|_{\mathcal{X}} \\
& =\max \left\{\lambda^{\prime}, \bar{\eta}\right\}\|S(t-x-h) \vartheta(\cdot)\|_{\mathcal{X}} \\
& \leq \max \left\{\lambda^{\prime}, \bar{\eta}\right\}\|\vartheta\| \\
& \left|P_{2}(0, t-x-h)\right| \leq \lambda\left|\vartheta_{0} e^{-\left(\lambda+\lambda^{\prime}\right)(t-x-h)}\right| \leq \lambda\|\vartheta\|, \\
& \left|P_{3}(0, t-x-h)\right| \leq \lambda \int_{0}^{\infty}\left|P_{1}(s, t-x-h)\right| d s \leq \lambda\|P(\cdot, t-x-h)\|_{\mathcal{X}} \\
& =\lambda\|S(t-x-h) \vartheta(\cdot)\|_{\mathcal{X}} \leq \lambda\|\vartheta\| .
\end{aligned}
$$

From (4.17)-(4.19) we can estimate first, third, and fifth term of (4.16) as follows:

$$
\begin{aligned}
& \int_{0}^{t}\left|P_{1}(0, t-x-h)\right|\left|e^{-\lambda(x+h)-\int_{0}^{x+h} \varphi(\tau) d \tau}-e^{-\lambda x-\int_{0}^{x} \varphi(\tau) d \tau}\right| d x \\
& \quad \leq \max \left\{\lambda^{\prime}, \bar{\eta}\right\}\|\vartheta\| \int_{0}^{t}\left|e^{-\lambda(x+h)-\int_{0}^{x+h} \varphi(\tau) d \tau}-e^{-\lambda x-\int_{0}^{x} \varphi(\tau) d \tau}\right| d x \\
& \quad \rightarrow 0, \quad \text { as }|h| \rightarrow 0, \text { uniformly for } \vartheta \\
& \int_{0}^{t}\left|P_{j}(0, t-x-h)\right|\left|e^{-\int_{0}^{x+h} \eta(\tau) d \tau}-e^{-\int_{0}^{x} \eta(\tau) d \tau}\right| d x \\
& \quad \leq \lambda\|\vartheta\| \int_{0}^{t}\left|e^{-\int_{0}^{x+h} \eta(\tau) d \tau}-e^{-\int_{0}^{x} \eta(\tau) d \tau}\right| d x \\
& \quad \rightarrow 0, \quad \text { as }|h| \rightarrow 0, \text { uniformly for } \vartheta, j=2,3 .
\end{aligned}
$$

Similarly, using the boundary condition and noting that $\eta(x)$ are Lipschitz continuous (without loss of generality assume that the Lipschitz constant is equal to 1 ), we have

$$
\begin{aligned}
& \left|P_{1}(0, t-x-h)-P_{1}(0, t-x)\right| \\
& \quad \leq\left|\lambda^{\prime} P_{0}(t-x-h)-\lambda^{\prime} P_{0}(t-x)\right|
\end{aligned}
$$




$$
\begin{aligned}
&+\left|\int_{0}^{\infty} P_{3}(s, t-x-h) \eta(s) d s-\int_{0}^{\infty} P_{3}(s, t-x) \eta(s) d s\right| \\
& \leq \lambda^{\prime}\left|\vartheta_{0} e^{-\left(\lambda+\lambda^{\prime}\right)(t-x-h)}-\vartheta_{0} e^{-\left(\lambda+\lambda^{\prime}\right)(t-x)}\right| \\
&+\mid \int_{0}^{t-x-h} P_{3}(s, t-x-h) \eta(s) d s+\int_{t-x-h}^{\infty} P_{3}(s, t-x-h) \eta(s) d s \\
&-\int_{0}^{t-x} P_{3}(s, t-x) \eta(s) d s-\int_{t-x}^{\infty} P_{3}(s, t-x) \eta(s) d s \mid \\
& \stackrel{t-x-h-s=z, t-x-s=z}{\underline{-}}
\end{aligned}
$$

\section{$\stackrel{t-x-h-s=z, t-x-s=z}{\underline{\underline{.}}}$}

$$
\begin{aligned}
\lambda^{\prime} \mid \vartheta_{0} & e^{-\left(\lambda+\lambda^{\prime}\right)(t-x-h)}-\vartheta_{0} e^{-\left(\lambda+\lambda^{\prime}\right)(t-x)} \mid \\
& +\mid \int_{0}^{t-x-h} P_{3}(t-x-h-z, t-x-h) \eta(t-x-h-z) d z \\
& +\int_{t-x-h}^{\infty} \vartheta_{3}(s-t+x+h) \eta(s) e^{-\int_{s-t+x+h}^{s} \eta(\tau) d \tau} d s \\
& -\int_{0}^{t-x} P_{3}(t-x-z, t-x) \eta(t-x-z) d z \\
& -\int_{t-x}^{\infty} \vartheta_{3}(s-t+x) \eta(s) e^{-\int_{s-t+x}^{s} \eta(\tau) d \tau} d s \mid \\
\leq & \lambda^{\prime}\left|\vartheta_{0}\right|\left|e^{-\left(\lambda+\lambda^{\prime}\right)(t-x-h)}-e^{-\left(\lambda+\lambda^{\prime}\right)(t-x)}\right| \\
& +\mid \int_{0}^{t-x-h} P_{3}(t-x-h-z, t-x-h) \eta(t-x-h-z) d z \\
& -\int_{0}^{t-x} P_{3}(t-x-z, t-x) \eta(t-x-z) d z \mid \\
& +\mid \int_{t-x-h}^{\infty} \vartheta_{3}(s-t+x+h) \eta(s) e^{-\int_{s-t+x+h}^{s} \eta(\tau) d \tau} d s \\
\hline & \int_{t-x}^{\infty} \vartheta_{3}(s-t+x) \eta(s) e^{-\int_{s-t+x}^{s} \eta(\tau) d \tau} d s \mid
\end{aligned}
$$

$$
\begin{aligned}
\lambda^{\prime}\left|\vartheta_{0}\right| & \left|e^{-\left(\lambda+\lambda^{\prime}\right)(t-x-h)}-e^{-\left(\lambda+\lambda^{\prime}\right)(t-x)}\right| \\
& +\mid \int_{0}^{t-x-h} \eta(t-x-h-z) P_{3}(0, z) e^{-\int_{0}^{t-x-h-z} \eta(\tau) d \tau} d z \\
& -\int_{0}^{t-x} \eta(t-x-z) P_{3}(0, z) e^{-\int_{0}^{t-x-z} \eta(\tau) d \tau} d z \mid \\
& +\mid \int_{0}^{\infty} \vartheta_{3}(z) \eta(z+t-x-h) e^{-\int_{z}^{z+t-x-h} \eta(\tau) d \tau} d z \\
& -\int_{0}^{\infty} \vartheta_{3}(z) \eta(z+t-x) e^{-\int_{z}^{z+t-x} \eta(\tau) d \tau} d z \mid \\
\leq & \lambda^{\prime}\left|\vartheta_{0}\right|\left|e^{-\left(\lambda+\lambda^{\prime}\right)(t-x-h)}-e^{-\left(\lambda+\lambda^{\prime}\right)(t-x)}\right| \\
& +\int_{t-x-h}^{t-x} \eta(t-x-h-z)\left|P_{3}(0, z)\right| e^{-\int_{0}^{t-x-h-z} \eta(\tau) d \tau} d z
\end{aligned}
$$




$$
\begin{aligned}
& +\int_{0}^{t-x}\left|P_{3}(0, z)\right||\eta(t-x-h-z)-\eta(t-x-z)| e^{-\int_{0}^{t-x-z} \eta(\tau) d \tau} d z \\
& +\int_{0}^{t-x} \eta(t-x-z)\left|P_{3}(0, z)\right|\left|e^{-\int_{0}^{t-x-h-z} \eta(\tau) d \tau}-e^{-\int_{0}^{t-x-z} \eta(\tau) d \tau}\right| d z \\
& +\left|\int_{0}^{\infty}\right| \vartheta_{3}(z)|| \eta(z+t-x-h)-\eta(z+t-x) \mid e^{-\int_{z}^{z+t-x-h} \eta(\tau) d \tau} d z \\
& -\int_{0}^{\infty} \eta(z+t-x)\left|\vartheta_{3}(z)\right|\left|e^{-\int_{z}^{z+t-x-h} \eta(\tau) d \tau}-e^{-\int_{z}^{z+t-x} \eta(\tau) d \tau}\right| d z \\
& \leq \lambda^{\prime}\left|\vartheta_{0}\right|\left|e^{-\left(\lambda+\lambda^{\prime}\right)(t-x-h)}-e^{-\left(\lambda+\lambda^{\prime}\right)(t-x)}\right| \\
& +\lambda\|\vartheta\|\left\{\bar{\eta}|h| \sup _{z \in[0, \infty)} e^{-\int_{0}^{t-x-h-z} \eta(\tau) d \tau}\right. \\
& +|h| \int_{0}^{t-x} e^{-\int_{0}^{t-x-h-z} \eta(\tau) d \tau} d z \\
& \left.+\bar{\eta} \int_{0}^{t-x}\left|e^{-\int_{0}^{t-x-h-z} \eta(\tau) d \tau}-e^{-\int_{0}^{t-x-z} \eta(\tau) d \tau}\right| d z\right\} \\
& +\|\vartheta\|\left\{|h| \sup _{z \in[0, \infty)} e^{-\int_{z}^{z+t-x-h} \eta(\tau) d \tau}\right. \\
& \left.+\bar{\eta} \sup _{z \in[0, \infty)}\left|e^{-\int_{z}^{z+t-x-h} \eta(\tau) d \tau}-e^{-\int_{z}^{z+t-x} \eta(\tau) d \tau}\right|\right\}
\end{aligned}
$$

$\rightarrow 0, \quad$ as $|h| \rightarrow 0$, uniformly for $\vartheta$

$$
\begin{aligned}
& \left|P_{2}(0, t-x-h)-P_{2}(0, t-x)\right| \\
& \quad \leq \lambda\left|\vartheta_{0} e^{-\left(\lambda+\lambda^{\prime}\right)(t-x-h)}-\vartheta_{0} e^{-\left(\lambda+\lambda^{\prime}\right)(t-x)}\right|
\end{aligned}
$$

$\rightarrow 0, \quad$ as $|h| \rightarrow 0$, uniformly for $\vartheta$

$$
\begin{aligned}
& \left|P_{3}(0, t-x-h)-P_{3}(0, t-x)\right| \\
& =\lambda\left|\int_{0}^{\infty} P_{1}(s, t-x-h) d s-\int_{0}^{\infty} P_{1}(s, t-x) d s\right| \\
& \leq \lambda \mid \int_{0}^{t-x-h} P_{1}(s, t-x-h) d s+\int_{t-x-h}^{\infty} P_{1}(s, t-x-h) d s \\
& \quad-\int_{0}^{t-x} P_{1}(s, t-x) d s-\int_{t-x}^{\infty} P_{1}(s, t-x) d s \mid
\end{aligned}
$$

\section{$\underline{\underline{t-x-h-s=z, t-x-s=z}}$}

$$
\begin{aligned}
& \lambda \mid \int_{0}^{t-x-h} P_{1}(t-x-h-z, t-x-h) d z \\
& \quad+\int_{t-x-h}^{\infty} \vartheta_{1}(s-t+x+h) e^{-\lambda(t-x-h)-\int_{s-t+x+h}^{s} \varphi(\tau) d \tau} d s \\
& \quad-\int_{0}^{t-x} P_{1}(t-x-z, t-x) d z \\
& \quad-\int_{t-x}^{\infty} \vartheta_{1}(s-t+x) e^{-\lambda(t-x)-\int_{s-t+x}^{s} \varphi(\tau) d \tau} d s \mid
\end{aligned}
$$




$$
\begin{aligned}
& \leq \lambda \mid \int_{0}^{t-x-h} P_{1}(t-x-h-z, t-x-h) d z \\
&-\int_{0}^{t-x} P_{1}(t-x-z, t-x) d z \mid \\
&+\lambda \mid \int_{t-x-h}^{\infty} \vartheta_{1}(s-t+x+h) e^{-\lambda(t-x-h)-\int_{s-t+x+h}^{s} \varphi(\tau) d \tau} d s \\
&-\int_{t-x}^{\infty} \vartheta_{1}(s-t+x) e^{-\lambda(t-x)-\int_{s-t+x}^{s} \varphi(\tau) d \tau} d s \mid \\
& s-t+x+h=z, s-t+x=z \\
& \hline
\end{aligned}
$$

$$
\begin{aligned}
& \lambda \mid \int_{0}^{t-x-h} P_{1}(0, z) e^{-\lambda(t-x-h)-\int_{0}^{t-x-h-z} \varphi(\tau) d \tau} d z \\
& -\int_{0}^{t-x} P_{1}(0, z) e^{-\lambda(t-x)-\int_{0}^{t-x-z} \varphi(\tau) d \tau} d z \\
& +\lambda \mid \int_{0}^{\infty} \vartheta_{1}(z) e^{-\lambda(t-x-h)-\int_{z}^{z+t-x-h} \varphi(\tau) d \tau} d z \\
& -\int_{0}^{\infty} \vartheta_{1}(z) e^{-\lambda(t-x)-\int_{z}^{z+t-x} \varphi(\tau) d \tau} d z \\
& \leq \lambda \int_{t-x-h}^{t-x}\left|P_{1}(0, z)\right| e^{-\lambda(t-x-h)-\int_{0}^{t-x-h-z} \varphi(\tau) d \tau} d z \\
& +\lambda \int_{0}^{t-x}\left|P_{1}(0, z)\right|\left|e^{-\lambda(t-x-h)-\int_{0}^{t-x-h-z} \varphi(\tau) d \tau}-e^{-\lambda(t-x)-\int_{0}^{t-x-z} \varphi(\tau) d \tau}\right| d z \\
& +\lambda \int_{0}^{\infty}\left|\vartheta_{1}(z)\right|\left|e^{-\lambda(t-x-h)-\int_{z}^{z+t-x-h} \varphi(\tau) d \tau}-e^{-\lambda(t-x)-\int_{z}^{z+t-x} \varphi(\tau) d \tau}\right| d z \\
& \leq \lambda \max \left\{\lambda^{\prime}, \bar{\eta}\right\}\|\vartheta\|\left\{|h| \sup _{z \in[0, \infty)} e^{-\lambda(t-x-h)-\int_{0}^{t-x-h-z} \varphi(\tau) d \tau}\right. \\
& \left.+\int_{0}^{t-x}\left|e^{-\lambda(t-x-h)-\int_{0}^{t-x-h-z} \varphi(\tau) d \tau}-e^{-\lambda(t-x)-\int_{0}^{t-x-z} \varphi(\tau) d \tau}\right| d z\right\} \\
& +\lambda\|\vartheta\| \sup _{z \in[0, \infty)}\left|e^{-\lambda(t-x-h)-\int_{z}^{z+t-x-h} \eta(\tau) d \tau}-e^{-\lambda(t-x)-\int_{z}^{z+t-x} \eta(\tau) d \tau}\right|
\end{aligned}
$$

$\rightarrow 0, \quad$ as $|h| \rightarrow 0$, uniformly for $\vartheta$

From (4.22)-(4.24) we deduce the estimation of the second, fourth, and sixth term of (4.16).

$$
\begin{aligned}
& \int_{0}^{t}\left|P_{1}(0, t-x-h)-P_{1}(0, t-x)\right|\left|e^{-\lambda x-\int_{0}^{x} \varphi(\tau) d \tau}\right| d x \\
& \quad \rightarrow 0, \quad \text { as }|h| \rightarrow 0, \text { uniformly for } \vartheta \\
& \int_{0}^{t}\left|P_{j}(0, t-x-h)-P_{j}(0, t-x)\right|\left|e^{-\int_{0}^{x} \eta(\tau) d \tau}\right| d x \\
& \quad \rightarrow 0, \quad \text { as }|h| \rightarrow 0, \text { uniformly for } \vartheta, j=2,3
\end{aligned}
$$


Combining (4.20)-(4.21) and (4.25)-(4.26) with (4.16), we conclude, for $x \in[0, t), h \in$ $(0, t], x+h \in[0, t)$,

$$
\sum_{i=1}^{3} \int_{0}^{t}\left|P_{i}(x+h, t)-P_{i}(x, t)\right| d x \quad \text { as }|h| \rightarrow 0 \text {, uniformly for } \vartheta .
$$

If $h \in[-t, 0), x+h \in[0, t)$, then, by using a similar argument, we can get the same result as (4.27), and the proof is finished.

Theorem 4.2 If the conditions of Theorem 4.1 hold, then $\mathbb{V}(t)$ satisfies

$$
\|\mathbb{V}(t) \vartheta\|_{\mathcal{X}} \leq e^{-\min \left\{\lambda+\lambda^{\prime}, \lambda+\underline{\varphi}, \underline{\eta}\right\} t}\|\vartheta\|_{\mathcal{X}}, \quad \forall \vartheta \in \mathcal{X}
$$

Proof For any $\vartheta \in \mathcal{X}$, from the definition of $\mathbb{V}(t)$ we estimate

$$
\begin{aligned}
\|\mathbb{V}(t) \vartheta(\cdot)\|_{\mathcal{X}}= & \left|\vartheta_{0} e^{-\left(\lambda+\lambda^{\prime}\right) t}\right|+\int_{t}^{\infty}\left|\vartheta_{1}(x-t) e^{-\lambda t-\int_{x-t}^{x} \varphi(\tau) d \tau}\right| d x \\
& +\int_{t}^{\infty}\left|\vartheta_{2}(x-t) e^{-\int_{x-t}^{x} \eta(\tau) d \tau}\right| d x+\int_{t}^{\infty}\left|\vartheta_{3}(x-t) e^{-\int_{x-t}^{x} \eta(\tau) d \tau}\right| d x \\
\leq & \left|\vartheta_{0}\right| e^{-\left(\lambda+\lambda^{\prime}\right) t}+e^{-(\lambda+\underline{\varphi}) t} \int_{t}^{\infty}\left|\vartheta_{1}(x-t)\right| d x \\
& +e^{-\underline{\eta} \underline{t}} \int_{t}^{\infty}\left|\vartheta_{2}(x-t)\right| d x+e^{-\underline{\eta} t} \int_{t}^{\infty}\left|\vartheta_{3}(x-t)\right| d x \\
= & \left|\vartheta_{0}\right| e^{-\left(\lambda+\lambda^{\prime}\right) t}+e^{-(\lambda+\underline{\varphi}) t}\left\|\vartheta_{1}\right\|_{L^{1}[0, \infty)} \\
& +e^{-\underline{\eta} \underline{t}}\left\|\vartheta_{2}\right\|_{L^{1}[0, \infty)}+e^{-\underline{\eta} t}\left\|\vartheta_{3}\right\|_{L^{1}[0, \infty)} \\
\leq & e^{-\min \left\{\lambda+\lambda^{\prime}, \lambda+\underline{\varphi}, \underline{\eta}\right] t}\|\vartheta\| \mathcal{X} .
\end{aligned}
$$

From Theorems 4.1 and 4.2 we get

$$
\|\mathbb{S}(t)-\mathbb{W}(t)\|=\|\mathbb{V}(t)\| \leq e^{-\min \left\{\lambda+\lambda^{\prime}, \lambda+\underline{\varphi}, \underline{\eta}\right] t} \rightarrow 0, \quad t \rightarrow \infty
$$

which together with the definition of quasi-compact operator (see Gupur [22, Definition 1.85]) allows us to obtain the following result.

Theorem 4.3 Under the same condition of Theorem 4.1, $\mathbb{S}(t)$ is a quasi-compact operator on $\mathcal{X}$.

Since $\mathbb{E}$ is a compact operator on $\mathcal{X}$, by Theorem 4.3 and Proposition 2.9 in Nagel [30, P. 215], we have the following result.

Corollary 4.1 Under the same condition of Theorem 4.1, $\mathbb{T}(t)$ is a quasi-compact operator on $\mathcal{X}$.

Consider now the spectral properties of the operator $\mathbb{A}+\mathbb{E}$.

Lemma 4.3 0 is an eigenvalue of $\mathbb{A}+\mathbb{E}$ with geometric multiplicity one. 
Proof Consider the equation $(\mathbb{A}+\mathbb{E}) \mathbf{P}=0$, i.e.,

$$
\begin{aligned}
& \left(\lambda+\lambda^{\prime}\right) P_{0}=\int_{0}^{\infty} \varphi(x) P_{1}(x) d x+\int_{0}^{\infty} \eta(x) P_{2}(x) d x, \\
& \frac{d P_{1}(x)}{d x}=-(\lambda+\varphi(x)) P_{1}(x), \\
& \frac{d P_{j}(x)}{d x}=-\eta(x) P_{j}(x), \quad j=1,2, \\
& P_{1}(0)=\lambda^{\prime} P_{0}+\int_{0}^{\infty} P_{3}(x) \eta(x) d x, \\
& P_{2}(0)=\lambda P_{0}, \\
& P_{3}(0)=\lambda \int_{0}^{\infty} P_{1}(x) d x .
\end{aligned}
$$

By solving (4.29)-(4.30), we have

$$
\begin{aligned}
& P_{1}(x)=\alpha_{1} e^{-\lambda x-\int_{0}^{x} \varphi(\tau) d \tau}, \\
& P_{j}(x)=\alpha_{j} e^{-\int_{0}^{x} \eta(\tau) d \tau}, \quad j=2,3 .
\end{aligned}
$$

Combining (4.34) and (4.35) with (4.28) and noting that $\int_{0}^{\infty} \eta(x) e^{-\int_{0}^{x} \eta(\tau) d \tau} d x=1$, we deduce

$$
\begin{aligned}
& \left(\lambda+\lambda^{\prime}\right) P_{0}=\alpha_{1} \int_{0}^{\infty} \varphi(x) e^{-\lambda x-\int_{0}^{x} \varphi(\tau) d \tau} d x+\lambda P_{0} \\
& \Longrightarrow \\
& \alpha_{1}=\frac{\lambda^{\prime}}{\int_{0}^{\infty} \varphi(x) e^{-\lambda x-\int_{0}^{x} \varphi(\tau) d \tau} d x} P_{0} .
\end{aligned}
$$

From (4.32)-(4.33) and (4.36) we have

$$
\begin{aligned}
\alpha_{2} & =\lambda P_{0}, \\
\alpha_{3} & =P_{3}(0)=\lambda a_{1} \int_{0}^{\infty} e^{-\lambda x-\int_{0}^{x} \varphi(\tau) d \tau} d x \\
& =\frac{\lambda \lambda^{\prime} \int_{0}^{\infty} e^{-\lambda x-\int_{0}^{x} \varphi(\tau) d \tau} d x}{\int_{0}^{\infty} \varphi(x) e^{-\lambda x-\int_{0}^{x} \varphi(\tau) d \tau} d x} P_{0} .
\end{aligned}
$$

Combining (4.34)-(4.35) with (4.36)-(4.38) yields

$$
\begin{aligned}
\|P\|= & \left|P_{0}\right|+\left\|P_{1}\right\|_{L^{1}[0, \infty)}+\left\|P_{2}\right\|_{L^{1}[0, \infty)}+\left\|P_{3}\right\|_{L^{1}[0, \infty)} \\
\leq & \left|P_{0}\right|+\left|\alpha_{1}\right| \int_{0}^{\infty} e^{-\lambda x-\int_{0}^{x} \varphi(\tau) d \tau} d x \\
& +\left|\alpha_{2}\right| \int_{0}^{\infty} e^{-\int_{0}^{x} \eta(\tau) d \tau} d x+\left|\alpha_{3}\right| \int_{0}^{\infty} e^{-\int_{0}^{x} \eta(\tau) d \tau} d x \\
\leq & \left|P_{0}\right|+\frac{\lambda^{\prime} \int_{0}^{\infty} e^{-\lambda x-\int_{0}^{x} \varphi(\tau) d \tau} d x}{\int_{0}^{\infty} \varphi(x) e^{-\lambda x-\int_{0}^{x} \varphi(\tau) d \tau} d x}\left|P_{0}\right|+\lambda \int_{0}^{\infty} e^{-\int_{0}^{x} \eta(\tau) d \tau} d x\left|P_{0}\right|
\end{aligned}
$$




$$
\begin{aligned}
& +\frac{\lambda \lambda^{\prime} \int_{0}^{\infty} e^{-\lambda x-\int_{0}^{x} \varphi(\tau) d \tau} d x \int_{0}^{\infty} e^{-\int_{0}^{x} \eta(\tau) d \tau} d x}{\int_{0}^{\infty} \varphi(x) e^{-\lambda x-\int_{0}^{x} \varphi(\tau) d \tau} d x}\left|P_{0}\right| \\
& <\infty
\end{aligned}
$$

This shows that 0 is an eigenvalue of $\mathbb{A}+\mathbb{E}$. Moreover, it is easy to see from (4.36)-(4.38) that the eigenvectors corresponding to the eigenvalue zero span a one-dimensional linear space, that is to say, the geometric multiplicity of 0 is one.

Lemma 4.4 $(\mathbb{A}+\mathbb{E})^{*}$ is given by

$$
(\mathbb{A}+\mathbb{E})^{*} \mathbf{Q}^{*}=(\mathbb{G}+\mathbb{F}) \mathbf{Q}^{*}, \quad \mathbf{Q}^{*} \in D\left((\mathbb{A}+\mathbb{E})^{*}\right)=D(\mathbb{G}),
$$

where

$$
\begin{aligned}
\mathbb{G} \mathbf{Q}^{*} & =\left(\begin{array}{cccc}
-\left(\lambda+\lambda^{\prime}\right) & 0 & 0 & 0 \\
0 & \frac{d}{d x}-(\lambda+\varphi(x)) & 0 & 0 \\
0 & 0 & \frac{d}{d x}-\eta(x) & 0 \\
0 & 0 & 0 & \frac{d}{d x}-\eta(x)
\end{array}\right)\left(\begin{array}{c}
Q_{0}^{*} \\
Q_{1}^{*}(x) \\
Q_{2}^{*}(x) \\
Q_{3}^{*}(x)
\end{array}\right), \\
\mathbb{F} \mathbf{Q}^{*} & =\left(\begin{array}{cccc}
0 & \lambda^{\prime} & \lambda & 0 \\
\varphi(x) & 0 & 0 & \lambda \\
\eta(x) & 0 & 0 & 0 \\
0 & \eta(x) & 0 & 0
\end{array}\right)\left(\begin{array}{c}
Q_{0}^{*} \\
Q_{1}^{*}(0) \\
Q_{2}^{*}(0) \\
Q_{3}^{*}(0)
\end{array}\right) \\
D(\mathbb{G}) & =\left\{\mathbf{Q}^{*} \in \mathcal{X}^{*} \mid \frac{d Q_{i}^{*}(x)}{d x} \text { exists and } Q_{i}^{*}(\infty)=\varepsilon(i=1,2,3)\right\}
\end{aligned}
$$

and $\varepsilon$ in $D(\mathbb{G})$ is a constant which is independent of $i$.

Lemma 4.5 0 is an eigenvalue of $(\mathbb{A}+\mathbb{E})^{*}$ with geometric multiplicity one.

Proof We consider the equation $(\mathbb{A}+\mathbb{E})^{*} \mathbf{Q}^{*}=0$, which is equivalent to

$$
\begin{aligned}
& -\left(\lambda+\lambda^{\prime}\right) Q_{0}^{*}+\lambda^{\prime} Q_{1}^{*}(0)+\lambda Q_{2}^{*}(0)=0, \\
& \frac{d Q_{1}^{*}(x)}{d x}-(\lambda+\varphi(x)) Q_{1}^{*}(x)+\varphi(x) Q_{0}^{*}+\lambda Q_{3}^{*}(0)=0, \\
& \frac{d Q_{2}^{*}(x)}{d x}-\eta(x) Q_{2}^{*}(x)+\eta(x) Q_{0}^{*}=0, \\
& \frac{d Q_{3}^{*}(x)}{d x}-\eta(x) Q_{3}^{*}(x)+\eta(x) Q_{1}^{*}(0)=0, \\
& Q_{1}^{*}(\infty)=Q_{2}^{*}(\infty)=Q_{3}^{*}(\infty)=\varepsilon .
\end{aligned}
$$

By solving (4.40)-(4.42) we deduce

$$
\begin{aligned}
Q_{1}^{*}(x)= & \beta_{1} e^{\int_{0}^{x}(\lambda+\varphi(\tau)) d \tau} \\
& -e^{\int_{0}^{x}(\lambda+\varphi(\tau)) d \tau} \int_{0}^{x}\left[\varphi(\xi) Q_{0}^{*}+\lambda Q_{3}^{*}(0)\right] e^{-\int_{0}^{\xi}(\lambda+\varphi(\tau)) d \tau} d \xi,
\end{aligned}
$$




$$
\begin{aligned}
Q_{2}^{*}(x)= & \beta_{2} e^{\int_{0}^{x} \eta(\tau) d \tau} \\
& -e^{\int_{0}^{x} \eta(\tau) d \tau} \int_{0}^{x} \eta(\xi) Q_{0}^{*} e^{-\int_{0}^{\xi} \eta(\tau) d \tau} d \xi, \\
Q_{3}^{*}(x)= & \beta_{3} e^{\int_{0}^{\xi} \eta(\tau) d \tau} \\
& -e^{\int_{0}^{x} \eta(\tau) d \tau} \int_{0}^{x} \eta(\xi) Q_{1}^{*}(0) e^{-\int_{0}^{\xi} \eta(\tau) d \tau} d \xi .
\end{aligned}
$$

Through multiplying the two sides of (4.44) by $e^{-\int_{0}^{x}(\lambda+\varphi(\tau)) d \tau}$ and multiplying $e^{-\int_{0}^{x} \eta(\tau) d \tau}$ by both sides of (4.45)-(4.46), we derive

$$
\begin{aligned}
& \beta_{1}=\int_{0}^{\infty}\left[\varphi(\xi) Q_{0}^{*}+\lambda Q_{3}^{*}(0)\right] e^{-\int_{0}^{\xi}(\lambda+\varphi(\tau)) d \tau} d \xi, \\
& \beta_{2}=\int_{0}^{\infty} \eta(\xi) Q_{0}^{*} e^{-\int_{0}^{\xi} \eta(\tau) d \tau} d \xi, \\
& \beta_{3}=\int_{0}^{\infty} \eta(\xi) Q_{1}^{*}(0) e^{-\int_{0}^{\xi} \eta(\tau) d \tau} d \xi .
\end{aligned}
$$

Substituting (4.47)-(4.49) into (4.44)-(4.46) separately, we have

$$
\begin{aligned}
Q_{1}^{*}(x) & =e^{\int_{0}^{x}(\lambda+\varphi(\tau)) d \tau} \int_{x}^{\infty}\left[\varphi(\xi) Q_{0}^{*}+\lambda Q_{3}^{*}(0)\right] e^{-\int_{0}^{\xi}(\lambda+\varphi(\tau)) d \tau} d \xi, \\
Q_{2}^{*}(x) & =Q_{0}^{*} e^{\int_{0}^{x} \eta(\tau) d \tau} \int_{x}^{\infty} \eta(\xi) e^{-\int_{0}^{\xi} \eta(\tau) d \tau} d \xi \\
& =Q_{0}^{*} e^{\int_{0}^{x} \eta(\tau) d \tau}\left(-\left.e^{-\int_{0}^{\xi} \eta(\tau) d \tau}\right|_{x} ^{\infty}\right)=Q_{0}^{*}, \\
Q_{3}^{*}(x) & =Q_{1}^{*}(0) e^{\int_{0}^{x} \eta(\tau) d \tau} \int_{x}^{\infty} \eta(\xi) e^{-\int_{0}^{\xi} \eta(\tau) d \tau} d \xi=Q_{1}^{*}(0) .
\end{aligned}
$$

On the other hand, inserting (4.50) into (4.39) we obtain

$$
\begin{aligned}
& -\left(\lambda+\lambda^{\prime}\right) Q_{0}^{*}+\lambda^{\prime} Q_{1}^{*}(0)+\lambda Q_{0}^{*}=0 \\
& \quad \Longrightarrow \\
& Q_{1}^{*}(0)=Q_{0}^{*} .
\end{aligned}
$$

Thus

$$
Q_{3}^{*}(0)=Q_{1}^{*}(0)=Q_{0}^{*}
$$

Substituting (4.52) into (4.50) we get

$$
\begin{aligned}
Q_{1}^{*}(x) & =Q_{0}^{*} e^{\int_{0}^{x}(\lambda+\varphi(\tau)) d \tau} \int_{x}^{\infty}[\lambda+\varphi(\xi)] e^{-\int_{0}^{\xi}(\lambda+\varphi(\tau)) d \tau} d \xi \\
& =Q_{0}^{*} e^{\int_{0}^{x}(\lambda+\varphi(\tau)) d \tau}\left(-\left.e^{-\int_{0}^{\xi}(\lambda+\varphi(\tau)) d \tau}\right|_{x} ^{\infty}\right)=Q_{0}^{*} .
\end{aligned}
$$

Altogether we estimate

$$
\left\|\left|\mathbf{Q}^{*} \|\right|=\sup \left\{\left|Q_{0}^{*}\right|,\left\|Q_{1}^{*}\right\|_{L^{\infty}[0, \infty)},\left\|Q_{2}^{*}\right\|_{L^{\infty}[0, \infty)},\left\|Q_{3}^{*}\right\|_{L^{\infty}[0, \infty)}\right\}=\left|Q_{0}^{*}\right|<\infty,\right.
$$


which shows that 0 is an eigenvalue of $(\mathbb{A}+\mathbb{E})^{*}$. Moreover, from (4.53), (4.55), and (4.56) it is not difficult to see that the geometric multiplicity of 0 is one.

Combining Lemmas 4.3 and 4.5 with Theorem 3.3, we know that the algebraic multiplicity of 0 is one and the spectral bound of $\mathbb{A}+\mathbb{E}$ is zero, that is, $s(\mathbb{A}+\mathbb{E})=0$. Consequently, by combining Theorem 3.3, Lemma 4.3, Lemma 4.5, and Corollary 4.1 with [22, Theorem 1.90], we conclude the following result.

Theorem 4.4 If $\varphi(x)$ and $\eta(x)$ are Lipschitz continuous and satisfy

$$
0<\underline{\varphi} \leq \varphi(x) \leq \bar{\varphi}<\infty, \quad \underline{\eta} \leq \eta(x) \leq \bar{\eta}<\infty,
$$

then there exist a positive projection $\operatorname{Pr}$ of rank one and suitable constants $\delta>0, M \geq 0$ such that

$$
\|\mathbb{T}(t)-\mathbb{P r}\| \leq M e^{-\delta t}
$$

where $\operatorname{Pr}=\frac{1}{2 \pi i} \int_{\bar{\Gamma}}(z I-\mathbb{A}-\mathbb{E})^{-1} d z$ and $\bar{\Gamma}$ is a circle with center 0 and sufficiently small radius.

Remark 4.1 From Theorem 3.3, Corollary 4.1, and Lemma 4.3, we know that $\{\gamma \in \sigma(\mathbb{A}+$ $\mathbb{E}) \mid \operatorname{Re} \gamma=0\}=\{0\}$. In other words, all points on the imaginary axis except zero belong to the resolvent set of $\mathbb{A}+\mathbb{E}$. Therefore we can conclude that, under the same condition of Theorem 4.4, the time-dependent solution of system (2.4) strongly converges to its steady-state solution, i.e., $\lim _{t \rightarrow \infty} \mathbf{P}(x, t)=\left\langle\mathbf{Q}^{*}, \mathbf{P}(0)\right\rangle \mathbf{P}(x)$, where $\mathbf{Q}^{*}(x)$ and $\mathbf{P}(x)$ are the eigenvectors corresponding to 0 in Lemma 4.5 and 4.3 .

In the following, by investigating the growth bound of $\mathbb{T}(t)$ and determining the explicit expression of the project operator $\mathbb{P r}$, we provide the main results of this section.

Lemma 4.6 For $\gamma \in \rho(\mathbb{A}+\mathbb{E})$, we have

$$
(\gamma I-\mathbb{A}+\mathbb{E})^{-1}\left(\begin{array}{l}
\mathbb{Z}_{0} \\
\mathbb{Z}_{1} \\
\mathbb{Z}_{2} \\
\mathbb{Z}_{3}
\end{array}\right)=\left(\begin{array}{l}
\mathbb{y}_{0} \\
\mathbb{y}_{1} \\
\mathbb{y}_{2} \\
\mathbb{y}_{3}
\end{array}\right), \quad \forall \mathbb{Z} \in \mathcal{X},
$$

where

$$
\begin{aligned}
\mathbb{y}_{0}= & {\left[\lambda \int_{0}^{\infty} e^{-(\gamma+\lambda) x-\int_{0}^{x} \varphi(\tau) d \tau} \int_{0}^{x} \mathbb{Z}_{1}(\tau) e^{(\gamma+\lambda) \tau-\int_{0}^{\tau} \varphi(\xi) d \xi} d \tau d x\right.} \\
& \times \int_{0}^{\infty} \eta(x) e^{-\gamma x-\int_{0}^{x} \eta(\tau) d \tau} d x \int_{0}^{\infty} \varphi(x) e^{-(\gamma+\lambda) x-\int_{0}^{x} \varphi(\tau) d \tau} d x \\
& +\int_{0}^{\infty} \eta(x) e^{-\gamma x-\int_{0}^{x} \eta(\tau) d \tau} \int_{0}^{x} \mathbb{Z}_{3}(\tau) e^{\gamma \tau-\int_{0}^{\tau} \eta(\xi) d \xi} d \tau d x \\
& \times \int_{0}^{\infty} \varphi(x) e^{-(\gamma+\lambda) x-\int_{0}^{x} \varphi(\tau) d \tau} d x
\end{aligned}
$$




$$
\begin{aligned}
& +\left(\int_{0}^{\infty} \varphi(x) e^{-(\gamma+\lambda) x-\int_{0}^{x} \varphi(\tau) d \tau} \int_{0}^{x} \mathbb{Z}_{1}(\tau) e^{(\gamma+\lambda) \tau-\int_{0}^{\tau} \varphi(\xi) d \xi} d \tau d x\right. \\
& \left.+\int_{0}^{\infty} \eta(x) e^{-\gamma x-\int_{0}^{x} \eta(\tau) d \tau} \int_{0}^{x} \mathbb{Z}_{2}(\tau) e^{\gamma \tau-\int_{0}^{\tau} \eta(\xi) d \xi} d \tau d x+\mathbb{Z}_{0}\right) \\
& \left.\times\left\{1-\lambda \int_{0}^{\infty} e^{-(\gamma+\lambda) x-\int_{0}^{x} \varphi(\tau) d \tau} d x \int_{0}^{\infty} \eta(x) e^{-\gamma x-\int_{0}^{x} \eta(\tau) d \tau} d x\right\}\right] \\
& /\left[\left(\gamma+\lambda+\lambda^{\prime}-\lambda \int_{0}^{\infty} \eta(x) e^{-\gamma x-\int_{0}^{x} \eta(\tau) d \tau} d x\right)\right. \\
& \times\left\{1-\lambda \int_{0}^{\infty} e^{-(\gamma+\lambda) x-\int_{0}^{x} \varphi(\tau) d \tau} d x \int_{0}^{\infty} \eta(x) e^{-\gamma x-\int_{0}^{x} \eta(\tau) d \tau} d x\right\} \\
& \left.-\lambda^{\prime} \int_{0}^{\infty} \varphi(x) e^{-(\gamma+\lambda) x-\int_{0}^{x} \varphi(\tau) d \tau} d x\right] \\
& \mathbb{y}_{1}(x)=\frac{\lambda^{\prime} e^{-(\gamma+\lambda) x-\int_{0}^{x} \varphi(\tau) d \tau}}{1-\lambda \int_{0}^{\infty} e^{-(\gamma+\lambda) x-\int_{0}^{x} \varphi(\tau) d \tau} d x \int_{0}^{\infty} \eta(x) e^{-\gamma x-\int_{0}^{x} \eta(\tau) d \tau} d x} \mathbb{y}_{0} \\
& +\frac{\lambda \int_{0}^{\infty} e^{-(\gamma+\lambda) x-\int_{0}^{x} \varphi(\tau) d \tau} \int_{0}^{x} \mathbb{Z}_{1}(\tau) e^{(\gamma+\lambda) \tau-\int_{0}^{\tau} \varphi(\xi) d \xi} d \tau d x}{1-\lambda \int_{0}^{\infty} e^{-(\gamma+\lambda) x-\int_{0}^{x} \varphi(\tau) d \tau} d x \int_{0}^{\infty} \eta(x) e^{-\gamma x-\int_{0}^{x} \eta(\tau) d \tau} d x} \\
& \times \int_{0}^{\infty} \eta(x) e^{-\gamma x-\int_{0}^{x} \eta(\tau) d \tau} d x e^{-(\gamma+\lambda) x-\int_{0}^{x} \varphi(\tau) d \tau} \\
& +\frac{\int_{0}^{\infty} \eta(x) e^{-\gamma x-\int_{0}^{x} \eta(\tau) d \tau} \int_{0}^{x} \mathbb{Z}_{3}(\tau) e^{\gamma \tau-\int_{0}^{\tau} \eta(\xi) d \xi} d \tau d x}{1-\lambda \int_{0}^{\infty} e^{-(\gamma+\lambda) x-\int_{0}^{x} \varphi(\tau) d \tau} d x \int_{0}^{\infty} \eta(x) e^{-\gamma x-\int_{0}^{x} \eta(\tau) d \tau} d x} \\
& \times e^{-(\gamma+\lambda) x-\int_{0}^{x} \varphi(\tau) d \tau} \\
& +e^{-(\gamma+\lambda) x-\int_{0}^{x} \varphi(\tau) d \tau} \int_{0}^{x} \mathbb{Z}_{1}(\tau) e^{(\gamma+\lambda) \tau-\int_{0}^{\tau} \varphi(\xi) d \xi} d \tau, \\
& \mathbb{Y}_{2}(x)=\lambda e^{-\gamma x-\int_{0}^{x} \eta(\tau) d \tau} \mathbb{Y}_{0}+e^{-\gamma x-\int_{0}^{x} \eta(\tau) d \tau} \int_{0}^{x} \mathbb{Z}_{2}(\tau) e^{\gamma \tau-\int_{0}^{\tau} \eta(\xi) d \xi} d \tau, \\
& \mathbb{Y}_{3}(x)=\frac{\lambda \lambda^{\prime} \int_{0}^{\infty} e^{-(\gamma+\lambda) x-\int_{0}^{x} \varphi(\tau) d \tau} d x e^{-\gamma x-\int_{0}^{x} \eta(\tau) d \tau}}{1-\lambda \int_{0}^{\infty} e^{-(\gamma+\lambda) x-\int_{0}^{x} \varphi(\tau) d \tau} d x \int_{0}^{\infty} \eta(x) e^{-\gamma x-\int_{0}^{x} \eta(\tau) d \tau} d x} \mathbb{Y}_{0} \\
& +\frac{\lambda \int_{0}^{\infty} e^{-(\gamma+\lambda) x-\int_{0}^{x} \varphi(\tau) d \tau} \int_{0}^{x} \mathbb{Z}_{1}(\tau) e^{(\gamma+\lambda) \tau-\int_{0}^{\tau} \varphi(\xi) d \xi} d \tau d x}{1-\lambda \int_{0}^{\infty} e^{-(\gamma+\lambda) x-\int_{0}^{x} \varphi(\tau) d \tau} d x \int_{0}^{\infty} \eta(x) e^{-\gamma x-\int_{0}^{x} \eta(\tau) d \tau} d x} \\
& \times e^{-\gamma x-\int_{0}^{x} \eta(\tau) d \tau} \\
& +\frac{\lambda \int_{0}^{\infty} \eta(x) e^{-\gamma x-\int_{0}^{x} \eta(\tau) d \tau} \int_{0}^{x} \mathbb{Z}_{3}(\tau) e^{\gamma \tau-\int_{0}^{\tau} \eta(\xi) d \xi} d \tau d x}{1-\lambda \int_{0}^{\infty} e^{-(\gamma+\lambda) x-\int_{0}^{x} \varphi(\tau) d \tau} d x \int_{0}^{\infty} \eta(x) e^{-\gamma x-\int_{0}^{x} \eta(\tau) d \tau} d x} \\
& \times \int_{0}^{\infty} e^{-(\gamma+\lambda) x-\int_{0}^{x} \varphi(\tau) d \tau} d x e^{-\gamma x-\int_{0}^{x} \eta(\tau) d \tau} \\
& +e^{-\gamma x-\int_{0}^{x} \eta(\tau) d \tau} \int_{0}^{x} \mathbb{Z}_{3}(\tau) e^{\gamma \tau-\int_{0}^{\tau} \eta(\xi) d \xi} d \tau .
\end{aligned}
$$

Proof For any given $\mathbb{Z} \in \mathcal{X}$, we consider the equation $(\gamma I-\mathbb{A}-\mathbb{E}) \mathbb{y}=\mathbb{Z}$, that is,

$$
\left(\gamma+\lambda+\lambda^{\prime}\right) \mathbb{y}_{0}=\int_{0}^{\infty} \varphi(x) \mathbb{y}_{1}(x) d x+\int_{0}^{\infty} \eta(x) \mathbb{y}_{2}(x) d x+\mathbb{Z}_{0}
$$




$$
\begin{aligned}
& \frac{d \mathbb{y}_{1}(x)}{d x}=-(\gamma+\lambda+\varphi(x)) \mathbb{y}_{1}(x)+\mathbb{Z}_{1}(x), \\
& \frac{d \mathbb{y}_{j}(x)}{d x}=-(\gamma+\eta(x)) \mathbb{y}_{j}(x)+\mathbb{Z}_{j}(x), \quad j=2,3, \\
& \mathbb{y}_{1}(0)=\lambda^{\prime} \mathbb{y}_{0}+\int_{0}^{\infty} \eta(x) \mathbb{y}_{3}(x) d x, \\
& \mathbb{y}_{2}(0)=\lambda \mathbb{y}_{0}, \\
& \mathbb{y}_{3}(0)=\lambda \int_{0}^{\infty} \mathbb{y}_{1}(x) d x .
\end{aligned}
$$

By solving (4.56)-(4.57), we have

$$
\begin{aligned}
\mathbb{Y}_{1}(x)= & \mathbf{d}_{1} e^{-(\gamma+\lambda) x-\int_{0}^{x} \varphi(\tau) d \tau} \\
& +e^{-(\gamma+\lambda) x-\int_{0}^{x} \varphi(\tau) d \tau} \int_{0}^{x} \mathbb{Z}_{1}(\tau) e^{(\gamma+\lambda) \tau-\int_{0}^{\tau} \varphi(\xi) d \xi} d \tau, \\
\mathbb{Y}_{j}(x)= & \mathbf{d}_{j} e^{-\gamma x-\int_{0}^{x} \eta(\tau) d \tau} \\
& +e^{-\gamma x-\int_{0}^{x} \eta(\tau) d \tau} \int_{0}^{x} \mathbb{Z}_{j}(\tau) e^{\gamma \tau-\int_{0}^{\tau} \eta(\xi) d \xi} d \tau, \quad j=2,3 .
\end{aligned}
$$

Considering (4.61)-(4.62) together with (4.58)-(4.60), we get

$$
\begin{aligned}
\mathbf{d}_{1}= & \lambda^{\prime} \mathbb{y}_{0}+\mathbf{d}_{3} \int_{0}^{\infty} \eta(x) e^{-\gamma x-\int_{0}^{x} \eta(\tau) d \tau} d x \\
& +\int_{0}^{\infty} \eta(x) e^{-\gamma x-\int_{0}^{x} \eta(\tau) d \tau} d x \int_{0}^{x} \mathbb{Z}_{3}(\tau) e^{\gamma \tau-\int_{0}^{\tau} \eta(\xi) d \xi} d \tau d x, \\
\mathbf{d}_{2}= & \lambda \mathbb{Y}_{0}, \\
\mathbf{d}_{3}= & \lambda \mathbf{d}_{1} \int_{0}^{\infty} e^{-(\gamma+\lambda) x-\int_{0}^{x} \varphi(\sigma) d \sigma} d x \\
& +\lambda \int_{0}^{\infty} e^{-(\gamma+\lambda) x-\int_{0}^{x} \varphi(\sigma) d \sigma} \int_{0}^{x} \mathbb{Z}_{1}(\tau) e^{(\gamma+\lambda) \tau-\int_{0}^{\tau} \varphi(\sigma) d \sigma} d \tau d x .
\end{aligned}
$$

Substituting (4.65) into (4.63), we deduce

$$
\begin{aligned}
\mathbf{d}_{1}= & \lambda^{\prime} \mathbb{y}_{0}+\lambda \mathbf{d}_{1} \int_{0}^{\infty} e^{-(\gamma+\lambda) x-\int_{0}^{x} \varphi(\tau) d \tau} d x \int_{0}^{\infty} \eta(x) e^{-\gamma x-\int_{0}^{x} \eta(\tau) d \tau} d x \\
& +\lambda \int_{0}^{\infty} e^{-(\gamma+\lambda) x-\int_{0}^{x} \varphi(\tau) d \tau} \int_{0}^{x} \mathbb{Z}_{1}(\tau) e^{(\gamma+\lambda) \tau-\int_{0}^{\tau} \varphi(\xi) d \xi} d \tau d x \\
& \times \int_{0}^{\infty} \eta(x) e^{-\gamma x-\int_{0}^{x} \eta(\tau) d \tau} d x \\
& +\int_{0}^{\infty} \eta(x) e^{-\gamma x-\int_{0}^{x} \eta(\tau) d \tau} d x \int_{0}^{x} \mathbb{Z}_{3}(\tau) e^{\gamma \tau-\int_{0}^{\tau} \eta(\xi) d \xi} d \tau d x \\
\Longrightarrow & \lambda^{\prime} \\
\mathbf{d}_{1}= & \frac{1-\lambda \int_{0}^{\infty} e^{-(\gamma+\lambda) x-\int_{0}^{x} \varphi(\sigma) d \sigma} d x \int_{0}^{\infty} \eta(x) e^{-\gamma x-\int_{0}^{x} \eta(\tau) d \tau} d x}{1} \mathbb{Y}_{0}
\end{aligned}
$$


Kasim and Gupur Boundary Value Problems

(2020) 2020:71

Page 21 of 37

$$
\begin{aligned}
& +\frac{\lambda \int_{0}^{\infty} e^{-(\gamma+\lambda) x-\int_{0}^{x} \varphi(\sigma) d \sigma} \int_{0}^{x} \mathbb{Z}_{1}(\tau) e^{(\gamma+\lambda) \tau-\int_{0}^{\tau} \varphi(\sigma) d \sigma} d \tau d x}{1-\lambda \int_{0}^{\infty} e^{-(\gamma+\lambda) x-\int_{0}^{x} \varphi(\sigma) d \sigma} d x \int_{0}^{\infty} \eta(x) e^{-\gamma x-\int_{0}^{x} \eta(\tau) d \tau} d x} \\
& \times \int_{0}^{\infty} \eta(x) e^{-\gamma x-\int_{0}^{x} \eta(\tau) d \tau} d x \\
& +\frac{\int_{0}^{\infty} \eta(x) e^{-\gamma x-\int_{0}^{x} \eta(\tau) d \tau} \int_{0}^{x} \mathbb{Z}_{3}(\tau) e^{\gamma \tau-\int_{0}^{\tau} \eta(\sigma) d \sigma} d \tau d x}{1-\lambda \int_{0}^{\infty} e^{-(\gamma+\lambda) x-\int_{0}^{x} \varphi(\sigma) d \sigma} d x \int_{0}^{\infty} \eta(x) e^{-\gamma x-\int_{0}^{x} \eta(\tau) d \tau} d x} .
\end{aligned}
$$

Substituting (4.66) into (4.65), we derive

$$
\begin{aligned}
\mathbf{d}_{3}= & \frac{\lambda \lambda^{\prime} \int_{0}^{\infty} e^{-(\gamma+\lambda) x-\int_{0}^{x} \varphi(\tau) d \tau} d x}{1-\lambda \int_{0}^{\infty} e^{-(\gamma+\lambda) x-\int_{0}^{x} \varphi(\tau) d \tau} d x \int_{0}^{\infty} \eta(x) e^{-\gamma x-\int_{0}^{x} \eta(\tau) d \tau} d x} \mathbb{y}_{0} \\
& +\frac{\lambda \int_{0}^{\infty} e^{-(\gamma+\lambda) x-\int_{0}^{x} \varphi(\tau) d \tau} \int_{0}^{x} \mathbb{Z}_{1}(\tau) e^{(\gamma+\lambda) \tau-\int_{0}^{\tau} \varphi(\xi) d \xi} d \tau d x}{1-\lambda \int_{0}^{\infty} e^{-(\gamma+\lambda) x-\int_{0}^{x} \varphi(\tau) d \tau} d x \int_{0}^{\infty} \eta(x) e^{-\gamma x-\int_{0}^{x} \eta(\tau) d \tau} d x} \\
& +\frac{\lambda \int_{0}^{\infty} \eta(x) e^{-\gamma x-\int_{0}^{x} \eta(\tau) d \tau} \int_{0}^{x} \mathbb{Z}_{3}(\tau) e^{\gamma \tau-\int_{0}^{\tau} \eta(\xi) d \xi} d \tau d x}{1-\lambda \int_{0}^{\infty} e^{-(\gamma+\lambda) x-\int_{0}^{x} \varphi(\tau) d \tau} d x \int_{0}^{\infty} \eta(x) e^{-\gamma x-\int_{0}^{x} \eta(\tau) d \tau} d x} \\
& \times \int_{0}^{\infty} e^{-(\gamma+\lambda) x-\int_{0}^{x} \varphi(\tau) d \tau} d x .
\end{aligned}
$$

Combining (4.64), (4.66), and (4.61)-(4.62) with (4.55) yields

$$
\begin{aligned}
& \left(\gamma+\lambda+\lambda^{\prime}\right) \mathbb{y}_{0} \\
& =\int_{0}^{\infty} \varphi(x) \mathbb{y}_{1}(x) d x+\int_{0}^{\infty} \eta(x) \mathbb{y}_{2}(x) d x+\mathbb{Z}_{0} \\
& =\frac{\lambda^{\prime} \int_{0}^{\infty} \varphi(x) e^{-(\gamma+\lambda) x-\int_{0}^{x} \varphi(\tau) d \tau} d x}{1-\lambda \int_{0}^{\infty} e^{-(\gamma+\lambda) x-\int_{0}^{x} \varphi(\tau) d \tau} d x \int_{0}^{\infty} \eta(x) e^{-\gamma x-\int_{0}^{x} \eta(\tau) d \tau} d x} \mathbb{y}_{0} \\
& +\frac{\lambda \int_{0}^{\infty} e^{-(\gamma+\lambda) x-\int_{0}^{x} \varphi(\tau) d \tau} \int_{0}^{x} \mathbb{Z}_{1}(\tau) e^{(\gamma+\lambda) \tau-\int_{0}^{\tau} \varphi(\xi) d \xi} d \tau d x}{1-\lambda \int_{0}^{\infty} e^{-(\gamma+\lambda) x-\int_{0}^{x} \varphi(\tau) d \tau} d x \int_{0}^{\infty} \eta(x) e^{-\gamma x-\int_{0}^{x} \eta(\tau) d \tau} d x} \\
& \times \int_{0}^{\infty} \eta(x) e^{-\gamma x-\int_{0}^{x} \eta(\tau) d \tau} d x \int_{0}^{\infty} \varphi(x) e^{-(\gamma+\lambda) x-\int_{0}^{x} \varphi(\tau) d \tau} d x \\
& +\frac{\int_{0}^{\infty} \eta(x) e^{-\gamma x-\int_{0}^{x} \eta(\tau) d \tau} \int_{0}^{x} \mathbb{Z}_{3}(\tau) e^{\gamma \tau-\int_{0}^{\tau} \eta(\xi) d \xi} d \tau d x}{1-\lambda \int_{0}^{\infty} e^{-(\gamma+\lambda) x-\int_{0}^{x} \varphi(\tau) d \tau} d x \int_{0}^{\infty} \eta(x) e^{-\gamma x-\int_{0}^{x} \eta(\tau) d \tau} d x} \\
& \times \int_{0}^{\infty} \varphi(x) e^{-(\gamma+\lambda) x-\int_{0}^{x} \varphi(\tau) d \tau} d x \\
& +\int_{0}^{\infty} \varphi(x) e^{-(\gamma+\lambda) x-\int_{0}^{x} \varphi(\tau) d \tau} \int_{0}^{x} \mathbb{Z}_{1}(\tau) e^{(\gamma+\lambda) \tau-\int_{0}^{\tau} \varphi(\xi) d \xi} d \tau d x \\
& +\lambda y_{0} \int_{0}^{\infty} \eta(x) e^{-\gamma x-\int_{0}^{x} \eta(\tau) d \tau} d x \\
& +\int_{0}^{\infty} \eta(x) e^{-\gamma x-\int_{0}^{x} \eta(\tau) d \tau} \int_{0}^{x} \mathbb{Z}_{2}(\tau) e^{\gamma \tau-\int_{0}^{\tau} \eta(\xi) d \xi} d \tau d x+\mathbb{Z}_{0} \\
& \Longrightarrow \\
& {\left[\left(\gamma+\lambda+\lambda^{\prime}-\lambda \int_{0}^{\infty} \eta(x) e^{-\gamma x-\int_{0}^{x} \eta(\tau) d \tau} d x\right)\right.}
\end{aligned}
$$




$$
\begin{aligned}
& \times\left\{1-\lambda \int_{0}^{\infty} e^{-(\gamma+\lambda) x-\int_{0}^{x} \varphi(\tau) d \tau} d x \int_{0}^{\infty} \eta(x) e^{-\gamma x-\int_{0}^{x} \eta(\tau) d \tau} d x\right\} \\
& \left.-\lambda^{\prime} \int_{0}^{\infty} \varphi(x) e^{-(\gamma+\lambda) x-\int_{0}^{x} \varphi(\tau) d \tau} d x\right] \mathbb{y}_{0} \\
& =\lambda \int_{0}^{\infty} e^{-(\gamma+\lambda) x-\int_{0}^{x} \varphi(\tau) d \tau} \int_{0}^{x} \mathbb{Z}_{1}(\tau) e^{(\gamma+\lambda) \tau-\int_{0}^{\tau} \varphi(\xi) d \xi} d \tau d x \\
& \times \int_{0}^{\infty} \eta(x) e^{-\gamma x-\int_{0}^{x} \eta(\tau) d \tau} d x \int_{0}^{\infty} \varphi(x) e^{-(\gamma+\lambda) x-\int_{0}^{x} \varphi(\tau) d \tau} d x \\
& +\int_{0}^{\infty} \eta(x) e^{-\gamma x-\int_{0}^{x} \eta(\tau) d \tau} \int_{0}^{x} \mathbb{Z}_{3}(\tau) e^{\gamma \tau-\int_{0}^{\tau} \eta(\sigma) d \sigma} d \tau d x \\
& \times \int_{0}^{\infty} \varphi(x) e^{-(\gamma+\lambda) x-\int_{0}^{x} \varphi(\tau) d \tau} d x \\
& +\left(\int_{0}^{\infty} \varphi(x) e^{-(\gamma+\lambda) x-\int_{0}^{x} \varphi(\tau) d \tau} \int_{0}^{x} \mathbb{Z}_{1}(\tau) e^{(\gamma+\lambda) \tau-\int_{0}^{\tau} \varphi(\xi) d \xi} d \tau d x\right. \\
& \left.+\int_{0}^{\infty} \eta(x) e^{-\gamma x-\int_{0}^{x} \eta(\sigma) d \sigma} \int_{0}^{x} \mathbb{Z}_{2}(\tau) e^{\gamma \tau-\int_{0}^{\tau} \eta(\xi) d \xi} d \tau d x+\mathbb{Z}_{0}\right) \\
& \times\left\{1-\lambda \int_{0}^{\infty} e^{-(\gamma+\lambda) x-\int_{0}^{x} \varphi(\tau) d \tau} d x \int_{0}^{\infty} \eta(x) e^{-\gamma x-\int_{0}^{x} \eta(\tau) d \tau} d x\right\} \\
& \Longrightarrow \\
& \mathbb{y}_{0}=\left[\lambda \int_{0}^{\infty} e^{-(\gamma+\lambda) x-\int_{0}^{x} \varphi(\tau) d \tau} \int_{0}^{x} \mathbb{Z}_{1}(\tau) e^{(\gamma+\lambda) \tau-\int_{0}^{\tau} \varphi(\xi) d \xi} d \tau d x\right. \\
& \times \int_{0}^{\infty} \eta(x) e^{-\gamma x-\int_{0}^{x} \eta(\tau) d \tau} d x \int_{0}^{\infty} \varphi(x) e^{-(\gamma+\lambda) x-\int_{0}^{x} \varphi(\tau) d \tau} d x \\
& +\int_{0}^{\infty} \eta(x) e^{-\gamma x-\int_{0}^{x} \eta(\tau) d \tau} \int_{0}^{x} \mathbb{Z}_{3}(\tau) e^{\gamma \tau-\int_{0}^{\tau} \eta(\xi) d \xi} d \tau d x \\
& \times \int_{0}^{\infty} \varphi(x) e^{-(\gamma+\lambda) x-\int_{0}^{x} \varphi(\tau) d \tau} d x \\
& +\left(\int_{0}^{\infty} \varphi(x) e^{-(\gamma+\lambda) x-\int_{0}^{x} \varphi(\tau) d \tau} \int_{0}^{x} \mathbb{Z}_{1}(\tau) e^{(\gamma+\lambda) \tau-\int_{0}^{\tau} \varphi(\sigma) d \sigma} d \tau d x\right. \\
& \left.+\int_{0}^{\infty} \eta(x) e^{-\gamma x-\int_{0}^{x} \eta(\tau) d \tau} \int_{0}^{x} \mathbb{Z}_{2}(\tau) e^{\gamma \tau-\int_{0}^{\tau} \eta(\xi) d \xi} d \tau d x+\mathbb{Z}_{0}\right) \\
& \left.\times\left\{1-\lambda \int_{0}^{\infty} e^{-(\gamma+\lambda) x-\int_{0}^{x} \varphi(\tau) d \tau} d x \int_{0}^{\infty} \eta(x) e^{-\gamma x-\int_{0}^{x} \eta(\tau) d \tau} d x\right\}\right] \\
& /\left[\left(\gamma+\lambda+\lambda^{\prime}-\lambda \int_{0}^{\infty} \eta(x) e^{-\gamma x-\int_{0}^{x} \eta(\tau) d \tau} d x\right)\right. \\
& \times\left\{1-\lambda \int_{0}^{\infty} e^{-(\gamma+\lambda) x-\int_{0}^{x} \varphi(\tau) d \tau} d x \int_{0}^{\infty} \eta(x) e^{-\gamma x-\int_{0}^{x} \eta(\tau) d \tau} d x\right\} \\
& \left.-\lambda^{\prime} \int_{0}^{\infty} \varphi(x) e^{-(\gamma+\lambda) x-\int_{0}^{x} \varphi(\tau) d \tau} d x\right] \text {. }
\end{aligned}
$$

Substituting (4.64), (4.66), and (4.67) into (4.61)-(4.62) separately, we obtain the remaining results of this lemma. 
Theorem 4.5 If $\varphi(x)$ and $\eta(x)$ are Lipschitz continuous and satisfy

$$
0<\underline{\varphi} \leq \varphi(x) \leq \bar{\varphi}<\infty, \quad \underline{\eta} \leq \eta(x) \leq \bar{\eta}<\infty
$$

then the time-dependent solution of system (2.4) converges exponentially to its steady-state solution, i.e.,

$$
\|\mathbf{P}(\cdot, t)-\mathbf{P}(\cdot)\| \leq M e^{-\delta t}, \quad t>0
$$

Proof Theorem 4.1 and Theorem 4.2 imply

$$
\begin{aligned}
& \|\mathbb{S}(t)-\mathbb{W}(t)\|=\|\mathbb{V}(t)\| \leq e^{-\min \left\{\lambda+\lambda^{\prime}, \lambda+\underline{\varphi}, \underline{\eta}\right\} t} \\
& \Longrightarrow \\
& \ln \|\mathbb{S}(t)-\mathbb{W}(t)\|=\ln \|\mathbb{V}(t)\| \leq-\min \left\{\lambda+\lambda^{\prime}, \lambda+\underline{\varphi}, \underline{\eta}\right\} t \\
& \Longrightarrow \\
& \lim _{t \rightarrow \infty} \frac{\ln \|\mathbb{S}(t)-\mathbb{W}(t)\|}{t} \leq-\min \left\{\lambda+\lambda^{\prime}, \lambda+\underline{\varphi}, \underline{\eta}\right\} t .
\end{aligned}
$$

From this together with Proposition 2.10 in Engel and Nagel [31, P. 258] we know that $\omega_{\text {ess }}(\mathbb{S}(t))$ (i.e., $\omega_{\text {ess }}(\mathbb{A})$ ), the essential growth bound of $\mathbb{S}(t)$ (i.e., $\left.\mathbb{A}\right)$, satisfies

$$
\omega_{\mathrm{ess}}(\mathbb{S}(t)) \leq-\min \left\{\lambda+\lambda^{\prime}, \lambda+\underline{\varphi}, \underline{\eta}\right\} .
$$

Since $\mathbb{E}: \mathcal{X} \rightarrow \mathbb{R}^{4}$ is a compact operator, by Proposition 2.12 in [31, P. 258] we deduce

$$
\omega_{\mathrm{ess}}(\mathbb{A}+\mathbb{E})=\omega_{\mathrm{ess}}(\mathbb{T}(t))=\omega_{\mathrm{ess}}(\mathbb{S}(t)) \leq-\min \left\{\lambda+\lambda^{\prime}, \lambda+\underline{\varphi}, \underline{\eta}\right\} .
$$

Using this result together with Corollary 2.11 in Engel and Nagel [31, P. 258] and Theorem 4.1 , we obtain that 0 is a pole of $(\gamma I-\mathbb{A}-\mathbb{E})^{-1}$ of order 1 . Therefore, from Theorem 4.5, Lemma 4.6, and the residue theorem, we have

$$
\operatorname{Pr}\left(\begin{array}{c}
\mathbb{Z}_{0} \\
\mathbb{Z}_{1} \\
\mathbb{Z}_{2} \\
\mathbb{Z}_{3}
\end{array}\right)=\lim _{\gamma \rightarrow 0} \gamma(\gamma I-\mathbb{A}-\mathbb{E})^{-1}\left(\begin{array}{c}
\mathbb{Z}_{0} \\
\mathbb{Z}_{1}(x) \\
\mathbb{Z}_{2}(x) \\
\mathbb{Z}_{3}(x)
\end{array}\right)=\left(\begin{array}{c}
\lim _{\gamma \rightarrow 0} \gamma \mathbb{Y}_{0} \\
\lim _{\gamma \rightarrow 0} \gamma \mathbb{Y}_{1}(x) \\
\lim _{\gamma \rightarrow 0} \gamma \mathbb{Y}_{2}(x) \\
\lim _{\gamma \rightarrow 0} \gamma \mathbb{Y}_{3}(x)
\end{array}\right) .
$$

Now, we are able to determine the projection operator by calculating the above limit. Taking into account that

$$
\begin{aligned}
& \int_{0}^{\infty} \eta(x) e^{-\int_{0}^{x} \eta(\tau) d \tau} d x=-\left.e^{-\lambda x} e^{-\int_{0}^{x} \eta(\tau) d \tau}\right|_{x=0} ^{x=\infty}=1, \\
& \int_{0}^{\infty} \varphi(x) e^{-\lambda x-\int_{0}^{x} \varphi(\tau) d \tau} d x=1-\lambda \int_{0}^{\infty} e^{-\lambda x} e^{-\int_{0}^{x} \varphi(\tau) d \tau} d x
\end{aligned}
$$

and using L'Hospital's rule, we obtain

$$
\lim _{\gamma \rightarrow 0} \gamma /\left[\left(\gamma+\lambda+\lambda^{\prime}-\lambda \int_{0}^{\infty} \eta(x) e^{-\gamma x-\int_{0}^{x} \eta(\tau) d \tau} d x\right)\right.
$$


Kasim and Gupur Boundary Value Problems

(2020) 2020:71

Page 24 of 37

$$
\begin{aligned}
& \times\left\{1-\lambda \int_{0}^{\infty} e^{-(\gamma+\lambda) x-\int_{0}^{x} \varphi(\sigma) d \sigma} d x \int_{0}^{\infty} \eta(x) e^{-\gamma x-\int_{0}^{x} \eta(\tau) d \tau} d x\right\} \\
& \left.-\lambda^{\prime} \int_{0}^{\infty} \varphi(x) e^{-(\gamma+\lambda) x-\int_{0}^{x} \varphi(\tau) d \tau} d x\right] \\
& =\lim _{\gamma \rightarrow 0} 1 /\left[\left(1+\lambda \int_{0}^{\infty} x \eta(x) e^{-\gamma x-\int_{0}^{x} \eta(\tau) d \tau} d x\right)\right. \\
& \times\left\{1-\lambda \int_{0}^{\infty} e^{-(\gamma+\lambda) x-\int_{0}^{x} \varphi(\tau) d \tau} d x \int_{0}^{\infty} \eta(x) e^{-\gamma x-\int_{0}^{x} \eta(\tau) d \tau} d x\right\} \\
& +\left(\gamma+\lambda+\lambda^{\prime}-\lambda \int_{0}^{\infty} \eta(x) e^{-\gamma x-\int_{0}^{x} \eta(\tau) d \tau} d x\right) \\
& \times\left\{\lambda \int_{0}^{\infty} x e^{-(\gamma+\lambda) x-\int_{0}^{x} \varphi(\tau) d \tau} d x \int_{0}^{\infty} \eta(x) e^{-\gamma x-\int_{0}^{x} \eta(\tau) d \tau} d x\right. \\
& \left.+\lambda \int_{0}^{\infty} e^{-(\gamma+\lambda) x-\int_{0}^{x} \varphi(\tau) d \tau} d x \int_{0}^{\infty} x \eta(x) e^{-\gamma x-\int_{0}^{x} \eta(\tau) d \tau} d x\right\} \\
& \left.+\lambda^{\prime} \int_{0}^{\infty} x \varphi(x) e^{-(\gamma+\lambda) x-\int_{0}^{x} \varphi(\tau) d \tau} d x\right] \\
& =1 /\left[\left(1+\lambda \int_{0}^{\infty} x \eta(x) e^{-\int_{0}^{x} \eta(\tau) d \tau} d x\right)\left\{1-\lambda \int_{0}^{\infty} e^{-\lambda x-\int_{0}^{x} \varphi(\tau) d \tau} d x\right\}\right. \\
& +\lambda^{\prime}\left\{\int_{0}^{\infty} x(\lambda+\varphi(x)) e^{-\lambda x-\int_{0}^{x} \varphi(\tau) d \tau} d x\right. \\
& \left.\left.+\lambda \int_{0}^{\infty} e^{-\lambda x-\int_{0}^{x} \varphi(\tau) d \tau} d x \int_{0}^{\infty} x \eta(x) e^{-\int_{0}^{x} \eta(\tau) d \tau} d x\right\}\right] \\
& =\frac{1}{\left(1+\lambda \int_{0}^{\infty} x \eta(x) e^{-\int_{0}^{x} \eta(\tau) d \tau} d x\right) \int_{0}^{\infty}\left(\lambda^{\prime}+\varphi(x)\right) e^{-\lambda x-\int_{0}^{x} \varphi(\tau) d \tau} d x} \\
& \doteq \frac{1}{H} \text {. }
\end{aligned}
$$

By the Fubini theorem we can get

$$
\begin{aligned}
& \int_{0}^{\infty} \varphi(x) e^{-\lambda x-\int_{0}^{x} \varphi(\tau) d \tau} \int_{0}^{x} \mathbb{Z}_{1}(\tau) e^{\lambda \tau+\int_{0}^{\tau} \varphi(\xi) d \xi} d \tau d x \\
& \quad=\int_{0}^{\infty} \mathbb{Z}_{1}(\tau) e^{\lambda \tau-\int_{0}^{\tau} \varphi(\xi) d \xi} \int_{\tau}^{\infty} e^{-\lambda x} d\left\{-e^{-\int_{0}^{x} \varphi(\tau) d \tau}\right\} d \tau \\
& =\int_{0}^{\infty} \mathbb{Z}_{1}(\tau) e^{\lambda \tau-\int_{0}^{\tau} \varphi(\xi) d \xi}\left[-\left.e^{-\lambda x} e^{-\int_{0}^{x} \varphi(\xi) d \xi}\right|_{x=\tau} ^{x=\infty}-\lambda \int_{\tau}^{\infty} e^{-\lambda x} e^{-\int_{0}^{x} \varphi(\tau) d \tau} d x\right] d \tau \\
& =\int_{0}^{\infty} \mathbb{Z}_{1}(x) d x-\lambda \int_{0}^{\infty} \mathbb{Z}_{1}(\tau) e^{\lambda \tau-\int_{0}^{\tau} \varphi(\xi) d \xi} \int_{\tau}^{\infty} e^{-\lambda x} e^{-\int_{0}^{x} \varphi(\tau) d \tau} d x d \tau, \\
& \int_{0}^{\infty} \eta(x) e^{-\int_{0}^{x} \eta(\tau) d \tau} \int_{0}^{x} \mathbb{Z}_{j}(\tau) e^{\int_{0}^{\tau} \eta(\xi) d \xi} d \tau d x=\int_{0}^{\infty} \mathbb{Z}_{j}(x) d x, \quad j=2,3 .
\end{aligned}
$$

Using this we derive

$$
\begin{aligned}
\lim _{\gamma \rightarrow 0} \gamma \mathbb{Y}_{0} \\
\quad=\left[\left\{\lambda \int_{0}^{\infty} e^{-\lambda x-\int_{0}^{x} \varphi(\tau) d \tau} \int_{0}^{x} \mathbb{Z}_{1}(\tau) e^{\lambda \tau-\int_{0}^{\tau} \varphi(\xi) d \xi} d \tau d x\right.\right.
\end{aligned}
$$


Kasim and Gupur Boundary Value Problems

(2020) $2020: 71$

Page 25 of 37

$$
\begin{aligned}
& \left.+\int_{0}^{\infty} \eta(x) e^{-\int_{0}^{x} \eta(\tau) d \tau} d x \int_{0}^{x} \mathbb{Z}_{3}(\tau) e^{-\int_{0}^{\tau} \eta(\xi) d \xi} d \tau d x\right\} \\
& \times \int_{0}^{\infty} \varphi(x) e^{-\lambda x-\int_{0}^{x} \varphi(\tau) d \tau} d x \\
& +\left\{\int_{0}^{\infty} \varphi(x) e^{-\lambda x-\int_{0}^{x} \varphi(\tau) d \tau} \int_{0}^{x} \mathbb{Z}_{1}(\tau) e^{\lambda \tau-\int_{0}^{\tau} \varphi(\xi) d \xi} d \tau d x\right. \\
& \left.+\int_{0}^{\infty} \eta(x) e^{-\int_{0}^{x} \eta(\tau) d \tau} \int_{0}^{x} \mathbb{Z}_{2}(\tau) e^{-\int_{0}^{\tau} \eta(\xi) d \xi} d \tau d x+\mathbb{Z}_{0}\right\} \\
& \left.\times\left\{1-\lambda \int_{0}^{\infty} e^{-\lambda x-\int_{0}^{x} \varphi(\tau) d \tau} d x\right\}\right] / H \\
& =\left[\lambda \int_{0}^{\infty} e^{-\lambda x-\int_{0}^{x} \varphi(\tau) d \tau} \int_{0}^{x} \mathbb{Z}_{1}(\tau) e^{\lambda \tau-\int_{0}^{\tau} \varphi(\xi) d \xi} d \tau d x\right. \\
& \times \int_{0}^{\infty} \varphi(x) e^{-\lambda x-\int_{0}^{x} \varphi(\tau) d \tau} d x \\
& +\int_{0}^{\infty} \mathbb{Z}_{3}(x) d x \int_{0}^{\infty} \varphi(x) e^{-\lambda x-\int_{0}^{x} \varphi(\tau) d \tau} d x+\left\{\int_{0}^{\infty} \mathbb{Z}_{1}(x) d x\right. \\
& \left.-\lambda \int_{0}^{\infty} e^{-\lambda x-\int_{0}^{x} \varphi(\tau) d \tau} \int_{0}^{x} \mathbb{Z}_{1}(\tau) e^{\lambda \tau-\int_{0}^{\tau} \varphi(\xi) d \xi} d \tau d x\right\} \\
& \times \int_{0}^{\infty} \varphi(x) e^{-\lambda x-\int_{0}^{x} \varphi(\tau) d \tau} d x \\
& \left.+\left\{\int_{0}^{\infty} \mathbb{Z}_{2}(x) d x+\mathbb{Z}_{0}\right\} \int_{0}^{\infty} \varphi(x) e^{-\lambda x-\int_{0}^{x} \varphi(\tau) d \tau} d x\right] / H \\
& =\left[\left\{\mathbb{Z}_{0}+\int_{0}^{\infty} \mathbb{Z}_{1}(x) d x+\int_{0}^{\infty} \mathbb{Z}_{2}(x) d x+\int_{0}^{\infty} \mathbb{Z}_{3}(x) d x\right\}\right. \\
& \left.\times \int_{0}^{\infty} \varphi(x) e^{-\lambda x-\int_{0}^{x} \varphi(\tau) d \tau} d x\right] / H \\
& =\frac{\int_{0}^{\infty} \varphi(x) e^{-\lambda x-\int_{0}^{x} \varphi(\tau) d \tau} d x}{H} \doteq P_{0}, \\
& \lim _{\gamma \rightarrow 0} \gamma \mathbb{Y}_{1}(x) \\
& =\frac{\lambda^{\prime} e^{-\lambda x-\int_{0}^{x} \varphi(\tau) d \tau}}{1-\lambda \int_{0}^{\infty} e^{-\lambda x-\int_{0}^{x} \varphi(\tau) d \tau} d x} \lim _{\gamma \rightarrow 0} \gamma \mathbb{Y}_{0} \\
& =\frac{\lambda^{\prime} e^{-\lambda x-\int_{0}^{x} \varphi(\tau) d \tau}}{H} \doteq P_{1}(x), \\
& \lim _{\gamma \rightarrow 0} \gamma \mathbb{Y}_{2}(x) \\
& =\lambda e^{-\gamma x-\int_{0}^{x} \eta(\tau) d \tau} \lim _{\gamma \rightarrow 0} \gamma \mathbb{Y}_{0} \\
& =\frac{\lambda e^{-\gamma x-\int_{0}^{x} \eta(\tau) d \tau} \int_{0}^{\infty} \varphi(x) e^{-\lambda x-\int_{0}^{x} \varphi(\tau) d \tau} d x}{H} \\
& \doteq P_{2}(x) \text {, } \\
& \lim _{\gamma \rightarrow 0} \gamma \mathbb{Y}_{3}(x)
\end{aligned}
$$




$$
\begin{aligned}
& =\frac{\lambda \lambda^{\prime} e^{-\int_{0}^{x} \eta(\tau) d \tau} \int_{0}^{\infty} e^{-\lambda x-\int_{0}^{x} \varphi(\tau) d \tau} d x}{1-\lambda \int_{0}^{\infty} e^{-\lambda x-\int_{0}^{x} \varphi(\tau) d \tau} d x \int_{0}^{\infty} \eta(x) e^{-\int_{0}^{x} \eta(\tau) d \tau} d x} \lim _{\gamma \rightarrow 0} \gamma \mathbb{Y}_{0} \\
& =\frac{\lambda \lambda^{\prime} e^{-\int_{0}^{x} \eta(\tau) d \tau} \int_{0}^{\infty} e^{-\lambda x-\int_{0}^{x} \varphi(\tau) d \tau} d x}{H} \doteq P_{3}(x) .
\end{aligned}
$$

Combining (4.69)-(4.72) with Theorem 4.5, we obtain

$$
\operatorname{Pr} \mathbf{P}(0)=\mathbf{P}(x)
$$

From Theorem 3.3, (4.73), and Theorem 4.5, we conclude

$$
\begin{aligned}
\|\mathbf{P}(\cdot, t)-\mathbf{P}(\cdot)\| & =\|T(t) \mathbf{P}(0)-\operatorname{Pr} \mathbf{P}(0)\| \leq\|T(t)-\operatorname{Pr}\|\|\mathbf{P}(0)\| \\
& \leq M e^{-\delta t}\|\mathbf{P}(0)\|=M e^{-\delta t}, \quad t \geq 0
\end{aligned}
$$

which implies that the time-dependent solution of system (2.4) converges exponentially to its steady-state solution.

\section{Asymptotic behavior of some reliability indices}

In this section, we briefly discuss the asymptotic behavior of some reliability indices of system (2.4). Their proof is similar to [22, P. 256], and we omit the details. From Remark 4.1 we have

$$
\lim _{t \rightarrow \infty} P_{0}(t)=P_{0}, \quad \lim _{t \rightarrow \infty} \int_{0}^{\infty}\left|P_{i}(x, t)-P_{i}(x)\right| d x=0, \quad i=1,2,3,
$$

which implies

$$
\begin{aligned}
& \lim _{t \rightarrow \infty} \int_{0}^{\infty}\left|\eta(x) P_{1}(x, t)-\eta(x) P_{1}(x)\right| d x=0, \\
& \lim _{t \rightarrow \infty} \int_{0}^{\infty}\left|\varphi(x) P_{j}(x, t)-\varphi(x) P_{j}(x)\right| d x=0, \quad j=2,3 .
\end{aligned}
$$

From its definition, the time-dependent availability of the system is given by

$$
A(t)=P_{0}(t)+\int_{0}^{\infty} P_{1}(x, t) d x
$$

which together with (4.34), (4.36), and (5.1) yields

$$
\begin{aligned}
A & =\lim _{t \rightarrow \infty} A(t)=P_{0}+\int_{0}^{\infty} P_{1}(x) d x \\
& =\left\{1+\frac{\lambda^{\prime} \int_{0}^{\infty} e^{-\lambda x-\int_{0}^{x} \varphi(\tau) d \tau} d x}{\int_{0}^{\infty} \varphi(x) e^{-\lambda x-\int_{0}^{x} \varphi(\tau) d \tau} d x}\right\} P_{0} .
\end{aligned}
$$

For the time-dependent failure frequency of the system, we have

$$
m_{f}=\lim _{t \rightarrow \infty} m_{f}(t)=\lambda P_{0}+\lambda \int_{0}^{\infty} P_{1}(x) d x=\lambda A
$$


The time-dependent renewal of the system means that the state of the system returns to the initial states and is given by

$$
\begin{aligned}
m_{r} & =\lim _{t \rightarrow \infty} m_{r}(t)=\lim _{t \rightarrow \infty}\left\{\int_{0}^{\infty} \varphi(x) P_{1}(x, t) d x+\int_{0}^{\infty} \eta(x) P_{2}(x, t) d x\right\} \\
& =\int_{0}^{\infty} P_{1}(x) \varphi(x) d x+\int_{0}^{\infty} P_{2}(x) \eta(x) d x \\
& =\frac{\lambda^{\prime} \int_{0}^{\infty} \varphi(x) e^{-\lambda x-\int_{0}^{x} \varphi(\tau) d \tau} d x}{\int_{0}^{\infty} \varphi(x) e^{-\lambda x-\int_{0}^{x} \varphi(\tau) d \tau} d x} P_{0}+\lambda \int_{0}^{\infty} \eta(x) e^{-\int_{0}^{x} \eta(\tau) d \tau} d x P_{0} \\
& =\left(\lambda+\lambda^{\prime}\right) P_{0} .
\end{aligned}
$$

If we let the failure states 2,3 be the absorbing states, then we obtain the following new system:

$$
\begin{aligned}
& \frac{d \widetilde{P}_{0}(t)}{d t}=-\left(\lambda+\lambda^{\prime}\right) \widetilde{P}_{0}(t)+\int_{0}^{\infty} \widetilde{P}_{1}(x, t) \varphi(x) d x, \\
& \frac{\partial \widetilde{P}_{1}(x, t)}{\partial t}+\frac{\partial \widetilde{P}_{1}(x, t)}{\partial x}=-(\lambda+\varphi(x)) \widetilde{P}_{1}(x, t), \\
& \widetilde{P}_{1}(0, t)=\lambda^{\prime} \widetilde{P}_{0}(t) .
\end{aligned}
$$

Hence, the time-dependent reliability of the system, by a similar argument, converges to a constant number, i.e.,

$$
\begin{aligned}
\lim _{t \rightarrow \infty} R(t) & =\lim _{t \rightarrow \infty}\left\{\widetilde{\mathbf{P}}_{0}(t)+\int_{0}^{\infty} \widetilde{\mathbf{P}}_{1}(t, x) d x\right\} \\
& =\widetilde{\mathbf{P}}_{0}+\int_{0}^{\infty} \widetilde{\mathbf{P}}_{1}(x) d x=R .
\end{aligned}
$$

Remark 5.1 If we add the normalizing condition $P_{0}(t)+\sum_{n=1}^{3} \int_{0}^{\infty} P_{i}(t, x) d x=1$, then in the steady-state case we obtain the results in Govil [27]. In other words, our results imply the results in Govil [27].

\section{Numerical results}

In this section we execute numerical experiments in order to study how the timedependent reliability indices, such as system availability $A(t)$, the failure frequency of the system $m_{f}(t)$, and the renewal frequency of the system $m_{r}(t)$, are affected by the change of each system parameter. There are four system parameters $\lambda, \lambda^{\prime}, \eta(x)$, and $\varphi(x)$. First of all, for convenience of the analysis, we assume that the repair time of the system is gamma distributed with constant repair rates $\eta(x)=\mu, \varphi(x)=\mu^{\prime}$ and fix the system parameters at values $\lambda=0.0004, \lambda^{\prime}=0.0006, \mu=0.004, \mu^{\prime}=0.006$.

In Fig. 2 we depict the variation of the system availability (Fig. 2(a)), failure frequency of the system (Fig. 2(b)), renewal frequency of the system (Fig. 2(c)) with respect to time $t$ for different values of $\beta$ ( $\beta$ is another parameter of the gamma distribution). It shows that time-dependent availability and failure frequency of the system decrease fast as time increases in each case, and after a long run they become constant at some value. The renewal frequency of the system $m_{r}(t)$ increases rapidly at an early stage as time increases 


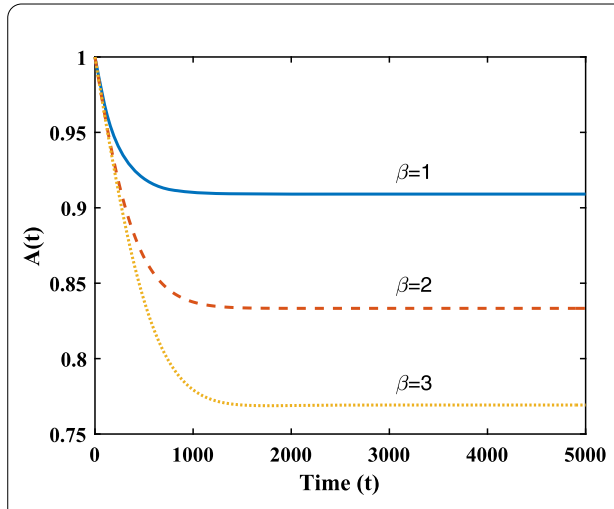

(a)

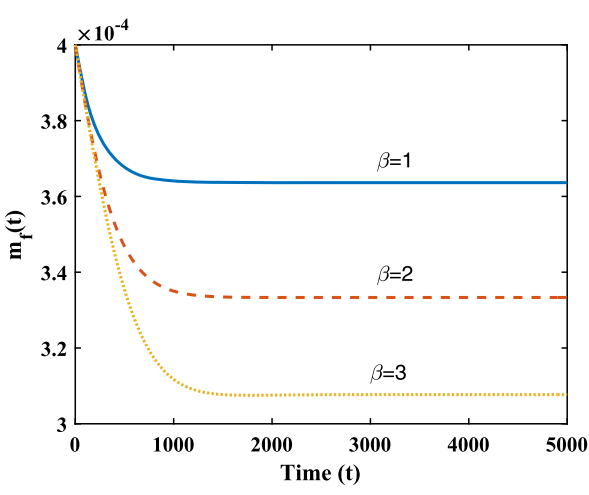

(b)

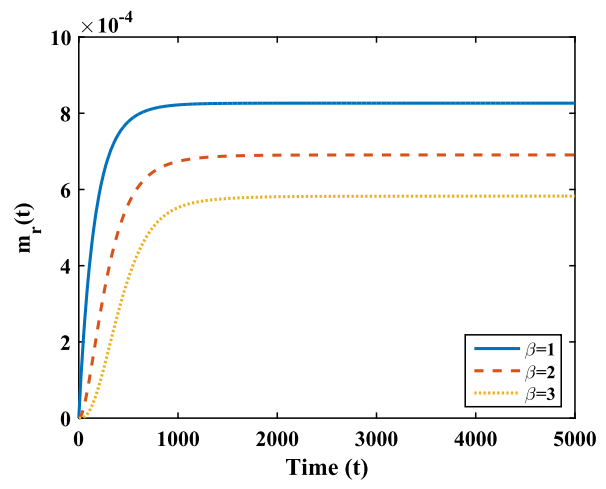

(c)

Figure 2 Time-dependent reliability indices for gamma distributed repair time. (a) Availability for different $\beta$; (b) Failure frequency for different $\beta$; (c) Renewal frequency for different $\beta$

and after a long run it becomes constant at some value. In addition, we also notice that system availability, failure frequency, and renewal frequency of the system decrease with increasing values of $\beta$.

In the following, we assume $\beta=1$ (i.e., the repair time of the system is exponentially distributed) and continue to pay attention to the effect on system availability, system failure frequency, and system renewal frequency of different values of the failure rates $\lambda$. Figure 3 reveals that all the indices converge to certain value as time grows and that, as $\lambda$ increases, the time-dependent system availability decreases, and failure frequency and renewal frequency increase.

Figure 4 shows the behavior of the system availability and the system renewal frequency for different repair rates, revealing that these indices increase as $\mu$ increases. Moreover, both indices approach a constant value as time goes to infinity.

The renewal frequency with different values of $\lambda^{\prime}$ and $\mu^{\prime}$ is represented in Fig. 5(a) and (b). From this figure, we can conclude that the renewal frequency of the system increases as $\lambda^{\prime}$ and $\mu^{\prime}$ increase. As shown, the renewal frequency tends to a constant value as time goes to infinity. Notice that the failure rate and repair rate of a component of Type II have no effect on the system availability.

Figure 6 shows the effect of $\lambda$ on the system reliability and mean time to failure (MTTF). We note that system reliability (Fig. 6(a)) and MTTF (Fig. 6(b)) decrease as $\lambda$ increases. Obviously, reliability vanishes as time goes to infinity. 


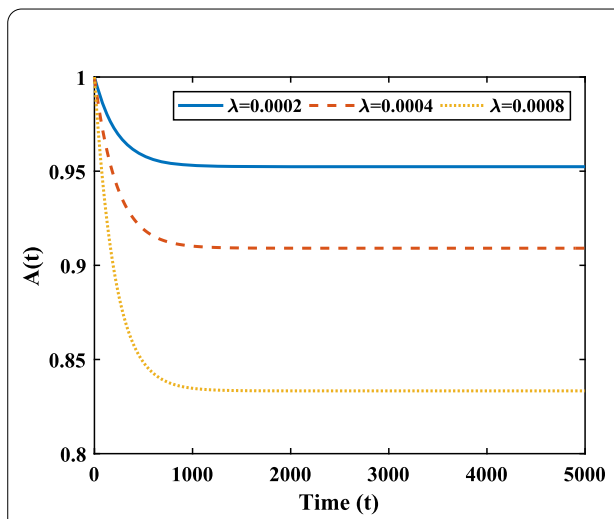

(a)

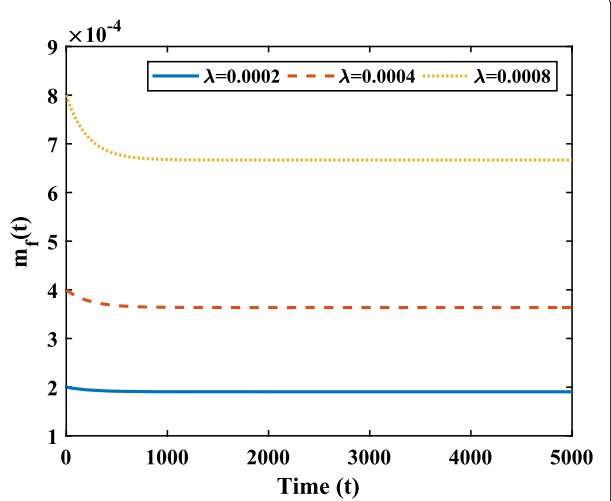

(b)

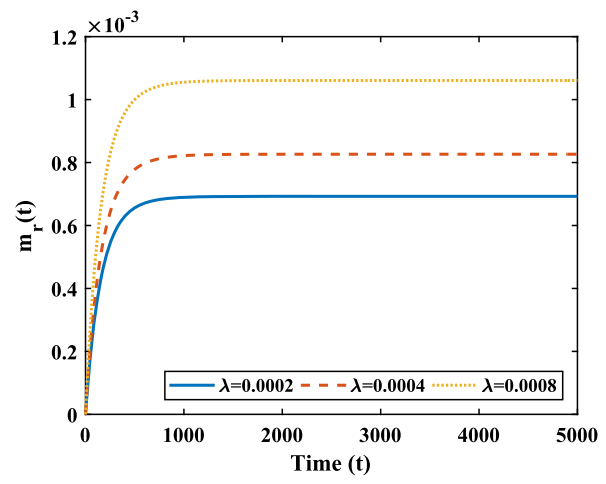

(c)

Figure 3 Time-dependent reliability indices with varying $\lambda$ for exponentially distributed repair time. (a) Availability for different $\lambda$; (b) Failure frequency for different $\lambda$; (c) Renewal frequency for different $\lambda$

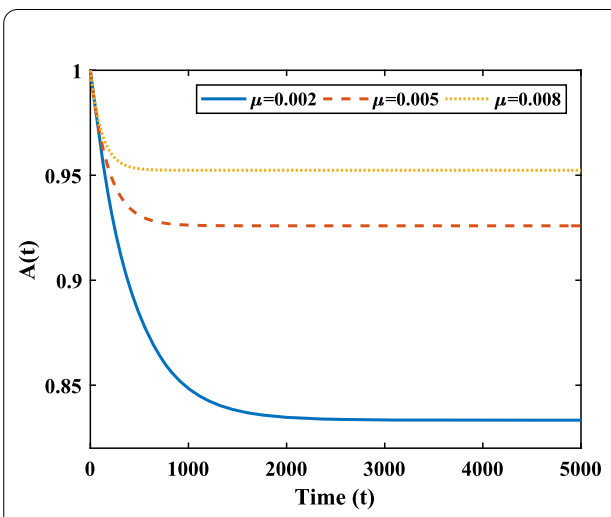

(a)

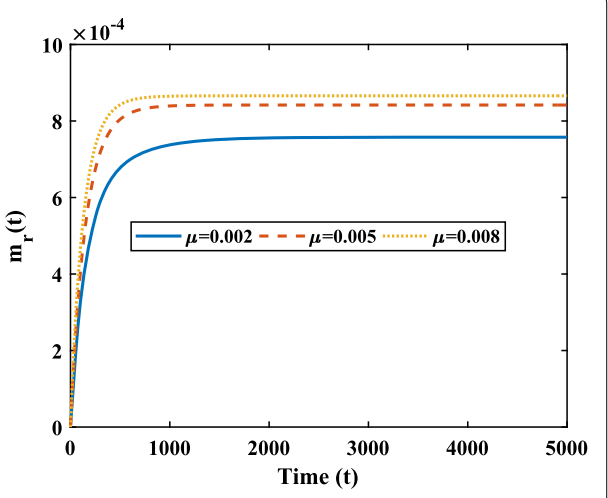

(b)

Figure 4 Effect of parameter $\mu$ on time-dependent reliability indices. (a) Availability for different $\mu$; (b) Renewal frequency for different $\mu$

In addition, all these numerical results show that the time-dependent reliability indices of the system converge to a constant value as time goes to infinity confirming the results obtained in Sect. 5. 


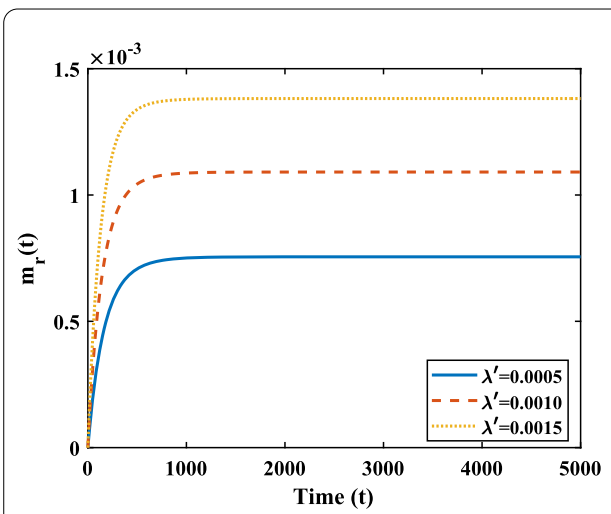

(a)

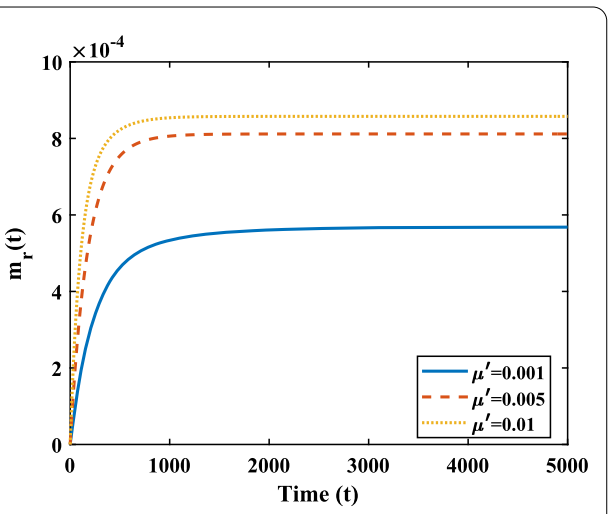

(b)

Figure 5 Effect of parameters $\lambda^{\prime}$ and $\mu^{\prime}$ on time-dependent renewal frequency. (a) Renewal frequency for different $\lambda^{\prime} ;(\mathbf{b})$ Renewal frequency for different $\mu^{\prime}$

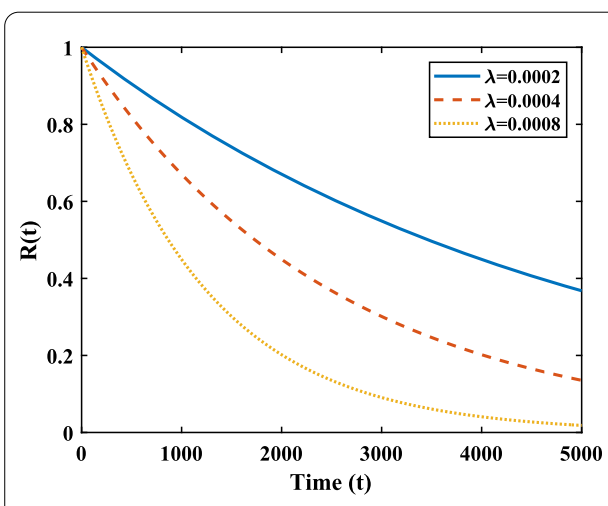

(a)

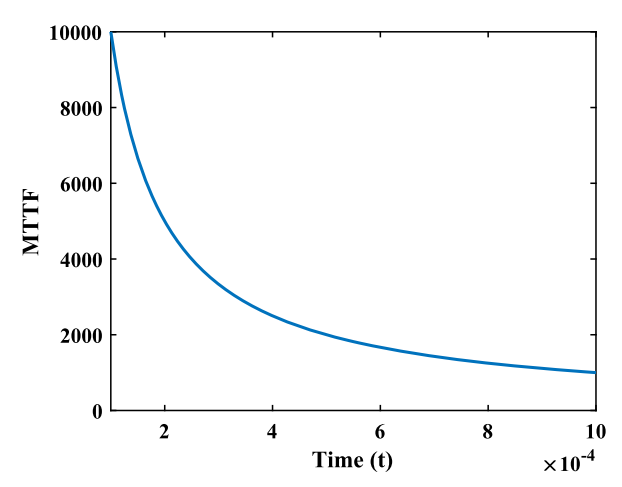

(b)

Figure 6 System reliability and MTTF for exponentially distributed repair time. (a) Reliability for different $\lambda$; (b) Effect of $\lambda$ on MTTF

\section{Conclusions}

This paper investigates the two-unit complex system, in which one of the components has priority with preemptive repeat repair disciplines. The model proposed is based on the supplementary variable technique and is described by partial differential equations with integral boundary conditions. We prove that the system is well-posed and that the time-dependent solution of the model converges exponentially to its steady-state solution. These results imply that the hypothesis mentioned in the Introduction holds for this model. Further, we show that the asymptotic behavior of some time-dependent reliability indices converges to constant numbers. In addition, some numerical examples are given to investigate the effects of changes in the parameters on system reliability indices.

The head-of-line repair discipline and preemptive resume repair discipline of the twounit complex system need to be considered in further studies. The effect of different repair priorities on the time-dependent reliability characteristics could also be an interesting topic for future studies. 


\section{Appendix}

Proof of Theorem 3.1 We split the proof into four steps. First of all, we estimate $\|(\gamma I-$ $\mathbb{A})^{-1} \|$, next we verify that $D(\mathbb{A})$ is dense in $\mathcal{X}$. Thirdly, we prove that $\mathbb{E}$ is a bounded linear operator. Lastly, we show that $\mathbb{A}+E$ is a dispersive operator, and therefore we obtain the desired result.

For any given $\Phi \in \mathcal{X}$, we consider the equation $(\gamma I-\mathbb{A}) \mathbf{P}=\Phi$, that is,

$$
\begin{aligned}
& \left(\gamma+\lambda+\lambda^{\prime}\right) P_{0}=\Phi_{0}, \\
& \frac{d P_{1}(x)}{d x}+(\gamma+\lambda+\varphi(x)) P_{1}(x)=\Phi_{1}(x), \\
& \frac{d P_{j}(x)}{d x}+(\gamma+\eta(x)) P_{j}(x)=\Phi_{j}(x), \quad j=2,3 \\
& P_{1}(0)=\lambda^{\prime} P_{0}+\int_{0}^{\infty} P_{3}(x) \eta(x) d x, \\
& P_{2}(0)=\lambda P_{0}, \\
& P_{3}(0)=\lambda \int_{0}^{\infty} P_{1}(x) d x .
\end{aligned}
$$

By solving (A.1)-(A.4), we have

$$
\begin{aligned}
& P_{0}=\frac{1}{\gamma+\lambda+\lambda^{\prime}} \Phi_{0}, \\
& P_{1}(x)=a_{1} e^{-(\gamma+\lambda) x-\int_{0}^{x} \varphi(\tau) d \tau}+e^{-(\gamma+\lambda) x-\int_{0}^{x} \varphi(\tau) d \tau} \int_{0}^{x} \Phi_{1}(\tau) e^{(\gamma+\lambda) \tau+\int_{0}^{\tau} \varphi(\sigma) d \sigma} d \tau, \\
& P_{j}(x)=a_{j} e^{-\gamma x-\int_{0}^{x} \eta(\tau) d \tau}+e^{-\gamma x-\int_{0}^{x} \eta(\tau) d \tau} \int_{0}^{x} \Phi_{j}(\tau) e^{\gamma \tau+\int_{0}^{\tau} \eta(\sigma) d \sigma} d \tau . j=2,3 .
\end{aligned}
$$

Considering (A.4)-(A.6) together with (A.7)-(A.9), we get

$$
\begin{aligned}
a_{1}= & P_{1}(0)=\lambda^{\prime} P_{0}+\int_{0}^{\infty} P_{3}(x) \eta(x) d x \\
= & \frac{\lambda^{\prime}}{\gamma+\lambda+\lambda^{\prime}} \Phi_{0} \\
& +\int_{0}^{\infty}\left[a_{3} e^{-\gamma x-\int_{0}^{x} \eta(\tau) d \tau}+e^{-\gamma x-\int_{0}^{x} \eta(\tau) d \tau} \int_{0}^{x} \Phi_{3}(\tau) e^{\gamma \tau+\int_{0}^{\tau} \eta(\sigma) d \sigma} d \tau\right] \eta(x) d x \\
= & \frac{\lambda^{\prime}}{\gamma+\lambda+\lambda^{\prime}} \Phi_{0}+a_{3} \int_{0}^{\infty} \eta(x) e^{-\gamma x-\int_{0}^{x} \eta(\tau) d \tau} d x \\
& +\int_{0}^{\infty} \eta(x) e^{-\gamma x-\int_{0}^{x} \eta(\tau) d \tau} d x \int_{0}^{x} \Phi_{3}(\tau) e^{\gamma \tau+\int_{0}^{\tau} \eta(\sigma) d \sigma} d \tau d x, \\
a_{2}= & P_{2}(0)=\lambda P_{0}, \\
a_{3}= & P_{3}(0)=\lambda \int_{0}^{\infty} P_{1}(x) d x \\
= & \lambda a_{1} \int_{0}^{\infty} e^{-(\gamma+\lambda) x-\int_{0}^{x} \varphi(\tau) d \tau} d x
\end{aligned}
$$




$$
+\lambda \int_{0}^{\infty} e^{-(\gamma+\lambda) x-\int_{0}^{x} \varphi(\tau) d \tau} \int_{0}^{x} \Phi_{1}(\tau) e^{(\gamma+\lambda) \tau+\int_{0}^{\tau} \varphi(\sigma) d \sigma} d \tau d x
$$

From (A.10)-(A.12) we deduce

$$
\begin{aligned}
a_{1}= & \left(1-\lambda \int_{0}^{\infty} e^{-(\gamma+\lambda) x-\int_{0}^{x} \varphi(\tau) d \tau} d x \int_{0}^{\infty} \eta(x) e^{-\gamma x-\int_{0}^{x} \eta(\tau) d \tau} d x\right)^{-1}\left[\frac{\lambda^{\prime}}{\gamma+\lambda+\lambda^{\prime}} \Phi_{0}\right. \\
& +\lambda \int_{0}^{\infty} e^{-(\gamma+\lambda) x-\int_{0}^{x} \varphi(\tau) d \tau} \int_{0}^{x} \Phi_{1}(\tau) e^{(\gamma+\lambda) \tau+\int_{0}^{\tau} \varphi(\sigma) d \sigma} d \tau d x \\
& \times \int_{0}^{\infty} \eta(x) e^{-\gamma x-\int_{0}^{x} \eta(\tau) d \tau} d x \\
& \left.+\int_{0}^{\infty} \eta(x) e^{-\gamma x-\int_{0}^{x} \eta(\tau) d \tau} d x \int_{0}^{x} \Phi_{3}(\tau) e^{\gamma \tau+\int_{0}^{\tau} \eta(\sigma) d \sigma} d \tau d x\right] .
\end{aligned}
$$

Using the Fubini theorem, we can estimate (assume $\gamma>0$ )

$$
\begin{aligned}
& \left|a_{1}\right| \leq\left(1-\frac{\lambda \bar{\eta}}{\gamma(\gamma+\lambda)}\right)^{-1}\left[\frac{\lambda^{\prime}}{\gamma+\lambda+\lambda^{\prime}}\left|\Phi_{0}\right|\right. \\
& +\frac{\lambda \bar{\eta}}{\gamma} \int_{0}^{\infty} e^{-(\gamma+\lambda) x} \int_{0}^{x} \Phi_{1}(\tau) e^{(\gamma+\lambda) \tau} d \tau d x \\
& \left.+\bar{\eta} \int_{0}^{\infty} e^{-\gamma x} d x \int_{0}^{x} \Phi_{3}(\tau) e^{\gamma \tau} d \tau d x\right] \\
& =\left(1-\frac{\lambda \bar{\eta}}{\gamma(\gamma+\lambda)}\right)^{-1}\left[\frac{\lambda^{\prime}}{\gamma+\lambda+\lambda^{\prime}}\left|\Phi_{0}\right|\right. \\
& +\frac{\lambda \bar{\eta}}{\gamma} \int_{0}^{\infty} \Phi_{1}(\tau) e^{(\gamma+\lambda) \tau} \int_{\tau}^{\infty} e^{-(\gamma+\lambda) x} d x d \tau \\
& \left.+\bar{\eta} \int_{0}^{\infty} \Phi_{3}(\tau) e^{\gamma \tau} \int_{\tau}^{\infty} e^{-\gamma x} d x d \tau\right] \\
& =\left(1-\frac{\lambda \bar{\eta}}{\gamma(\gamma+\lambda)}\right)^{-1}\left[\frac{\lambda^{\prime}}{\gamma+\lambda+\lambda^{\prime}}\left|\Phi_{0}\right|\right. \\
& \left.+\frac{\lambda \bar{\eta}}{\gamma(\gamma+\lambda)} \int_{0}^{\infty} \Phi_{1}(\tau) d \tau+\frac{\bar{\eta}}{\gamma} \int_{0}^{\infty} \Phi_{3}(\tau) d \tau\right] \\
& =\frac{\gamma(\gamma+\lambda)}{\gamma(\gamma+\lambda)-\lambda \bar{\eta}} \frac{\lambda^{\prime}}{\gamma+\lambda+\lambda^{\prime}}\left|\Phi_{0}\right|+\frac{\lambda \bar{\eta}}{\gamma(\gamma+\lambda)-\lambda \bar{\eta}}\left\|\Phi_{1}\right\|_{L^{1}[0, \infty)} \\
& +\frac{\bar{\eta}(\gamma+\lambda)}{\gamma(\gamma+\lambda)-\lambda \bar{\eta}}\left\|\Phi_{3}\right\|_{L^{1}[0, \infty)} \\
& \left|a_{2}\right|=\lambda\left|P_{0}\right|=\frac{\lambda}{\gamma+\lambda+\lambda^{\prime}}\left|\Phi_{0}\right|, \\
& \left|a_{3}\right| \leq \frac{\lambda}{\gamma+\lambda}\left|a_{1}\right|+\frac{\lambda}{\gamma+\lambda} \int_{0}^{\infty}\left|\Phi_{1}(\tau)\right| d \tau \\
& \leq \frac{\lambda \gamma}{\gamma(\gamma+\lambda)-\lambda \bar{\eta}} \frac{\lambda^{\prime}}{\gamma+\lambda+\lambda^{\prime}}\left|\Phi_{0}\right|+\frac{\lambda \gamma}{\gamma(\gamma+\lambda)-\lambda \bar{\eta}}\left\|\Phi_{1}\right\|_{L^{1}[0, \infty)} \\
& +\frac{\lambda \bar{\eta}}{\gamma(\gamma+\lambda)-\lambda \bar{\eta}}\left\|\Phi_{3}\right\|_{L^{1}[0, \infty)}
\end{aligned}
$$


where we have used the following inequalities:

$$
e^{-\int_{0}^{x} \phi(\tau) d \tau} \leq 1, \quad e^{-\int_{0}^{x} \phi(\tau) d \tau} \leq 1, \quad x \geq 0
$$

Now, combining (A.14)-(A.16) with (A.7)-(A.9) and using the Fubini theorem, we calculate

$$
\begin{aligned}
\left\|P_{1}\right\|_{L^{1}[0, \infty)} & =\int_{0}^{\infty}\left|P_{1}(x)\right| d x \\
& \leq \int_{0}^{\infty}\left|a_{1}\right| e^{-(\gamma+\lambda) x d \tau} d x+\int_{0}^{\infty} e^{-(\gamma+\lambda) x} \int_{0}^{x}\left|\Phi_{1}(\tau)\right| e^{(\gamma+\lambda) \tau} d \tau d x \\
& \leq \frac{1}{\gamma+\lambda}\left|a_{1}\right|+\int_{0}^{\infty}\left|\Phi_{1}(\tau)\right| e^{(\gamma+\lambda) \tau} \int_{\tau}^{\infty} e^{-(\gamma+\lambda) x} d x d \tau \\
& =\frac{1}{\gamma+\lambda}\left|a_{1}\right|+\frac{1}{\gamma+\lambda}\left\|\Phi_{1}\right\|_{L^{1}[0, \infty)}, \\
\left\|P_{j}\right\|_{L^{1}[0, \infty)} & =\int_{0}^{\infty}\left|P_{j}(x)\right| d x \leq \frac{1}{\gamma}\left|a_{2}\right|+\frac{1}{\gamma}\left\|\Phi_{2}\right\|_{L^{1}[0, \infty)}, \quad j=2,3 .
\end{aligned}
$$

(A.14)-(A.18) give

$$
\begin{aligned}
& \|\mathbf{P}\|=\left|P_{0}\right|+\sum_{i=1}^{\infty}\left\|P_{i}\right\|_{L^{1}[0, \infty)} \\
& \leq \frac{1}{\gamma+\lambda+\lambda^{\prime}}\left|\Phi_{0}\right|+\frac{1}{\gamma+\lambda}\left[\frac{\gamma(\gamma+\lambda)}{\gamma(\gamma+\lambda)-\lambda \bar{\eta}} \frac{\lambda^{\prime}}{\gamma+\lambda+\lambda^{\prime}}\left|\Phi_{0}\right|\right. \\
& \left.+\frac{\lambda \bar{\eta}}{\gamma(\gamma+\lambda)-\lambda \bar{\eta}}\left\|\Phi_{1}\right\|_{L^{1}[0, \infty)}+\frac{\bar{\eta}(\gamma+\lambda)}{\gamma(\gamma+\lambda)-\lambda \bar{\eta}}\left\|\Phi_{3}\right\|_{L^{1}[0, \infty)}\right] \\
& +\frac{1}{\gamma+\lambda}\left\|\Phi_{1}\right\|_{L^{1}[0, \infty)}+\frac{\lambda}{\gamma\left(\gamma+\lambda+\lambda^{\prime}\right)}\left|\Phi_{0}\right|+\frac{1}{\gamma}\left\|\Phi_{2}\right\|_{L^{1}[0, \infty)} \\
& +\frac{1}{\gamma}\left[\frac{\lambda \gamma}{\gamma(\gamma+\lambda)-\lambda \bar{\eta}} \frac{\lambda^{\prime}}{\gamma+\lambda+\lambda^{\prime}}\left|\Phi_{0}\right|+\frac{\lambda \gamma}{\gamma(\gamma+\lambda)-\lambda \bar{\eta}}\left\|\Phi_{1}\right\|_{L^{1}[0, \infty)}\right. \\
& \left.+\frac{\lambda \bar{\eta}}{\gamma(\gamma+\lambda)-\lambda \bar{\eta}}\left\|\Phi_{3}\right\|_{L^{1}[0, \infty)}\right]+\frac{1}{\gamma}\left\|\Phi_{3}\right\|_{L^{1}[0, \infty)} \\
& \leq\left[\frac{1}{\gamma+\lambda+\lambda^{\prime}}+\frac{\gamma}{\gamma(\gamma+\lambda)-\lambda \bar{\eta}} \frac{\lambda^{\prime}}{\gamma+\lambda+\lambda^{\prime}}\right. \\
& \left.+\frac{\lambda}{\gamma\left(\gamma+\lambda+\lambda^{\prime}\right)}+\frac{\lambda}{\gamma(\gamma+\lambda)-\lambda \bar{\eta}} \frac{\lambda^{\prime}}{\gamma+\lambda+\lambda^{\prime}}\right]\left|\Phi_{0}\right| \\
& +\left[\frac{\lambda \bar{\eta}}{\gamma(\gamma+\lambda)-\lambda \bar{\eta}} \frac{1}{\gamma+\lambda}+\frac{1}{\gamma+\lambda}\right. \\
& \left.+\frac{\lambda}{\gamma(\gamma+\lambda)-\lambda \bar{\eta}}\right]\left\|\Phi_{1}\right\|_{L^{1}[0, \infty)}+\frac{1}{\gamma}\left\|\Phi_{2}\right\|_{L^{1}[0, \infty)} \\
& +\left[\frac{\bar{\eta}}{\gamma(\gamma+\lambda)-\lambda \bar{\eta}}+\frac{\lambda \bar{\eta}}{\gamma(\gamma(\gamma+\lambda)-\lambda \bar{\eta})}+\frac{1}{\gamma}\right]\left\|\Phi_{3}\right\|_{L^{1}[0, \infty)} \\
& =\frac{\gamma(\gamma+\lambda)\left(\gamma+\lambda+\lambda^{\prime}\right)-\lambda \bar{\eta}(\gamma+\lambda)}{\gamma[\gamma(\gamma+\lambda)-\lambda \bar{\eta}]\left(\gamma+\lambda+\lambda^{\prime}\right)}\left|\Phi_{0}\right|
\end{aligned}
$$




$$
\begin{aligned}
& +\frac{\gamma+\lambda}{\gamma(\gamma+\lambda)-\lambda \bar{\eta}}\left\|\Phi_{1}\right\|_{L^{1}[0, \infty)}+\frac{1}{\gamma}\left\|\Phi_{2}\right\|_{L^{1}[0, \infty)} \\
& +\frac{\gamma+\lambda+\bar{\eta}}{\gamma(\gamma+\lambda)-\lambda \bar{\eta}}\left\|\Phi_{3}\right\|_{L^{1}[0, \infty)} \\
\leq & \frac{1}{\gamma-\bar{\eta}}\|\Phi\| .
\end{aligned}
$$

(A.19) shows that $(\gamma I-\mathbb{A})^{-1}$ exists for $\gamma>\bar{\eta}$, and

$$
(\gamma I-\mathbb{A})^{-1}: \mathcal{X} \rightarrow D(\mathbb{A}), \quad\left\|(\gamma I-\mathbb{A})^{-1}\right\| \leq \frac{1}{\gamma-\bar{\eta}} .
$$

In the following, we should prove that $D(\mathbb{A})$ is dense in $\mathcal{X}$. The proof is similar to Gupur [22, P.76], and we omit the details. From the above results and Hille-Yosida theorem (see Nagel [30]), we conclude that $\mathbb{A}$ generates a $C_{0}$-semigroup. We now verify that $\mathbb{E}$ is a bounded linear operator. From the definition of $\mathbb{E}$ we have, for $\mathbf{P} \in \mathcal{X}$,

$$
\begin{aligned}
\|\mathbb{E} \mathbf{P}\| & \leq \int_{0}^{\infty}\left|\varphi(x) P_{1}(x)\right| d x+\int_{0}^{\infty}\left|\eta(x) P_{2}(x)\right| d x \\
& \leq \bar{\varphi} \int_{0}^{\infty}\left|P_{1}(z)\right| d z+\bar{\eta} \int_{0}^{\infty}\left|P_{2}(x)\right| d x \\
& \leq \max \{\bar{\varphi}, \bar{\eta}\}\|\mathbf{P}\| .
\end{aligned}
$$

Expression (A.20) implies that $\mathbb{E}$ is a bounded operator and it is not difficult to show that it is also linear. Hence, from the perturbation theory of the $C_{0}$-semigroup [22, Theorem 1.80], we get that $\mathbb{A}+\mathbb{E}$ generates a $C_{0}$-semigroup $T(t)$. Finally, we prove that $\mathbb{A}+\mathbb{E}$ is a dispersive operator. For $\mathbf{P} \in D(\mathbb{A})$, we choose $\boldsymbol{\xi}$, where

$$
\xi(x)=\left(\frac{\left[P_{0}\right]^{+}}{P_{0}}, \frac{\left[P_{1}(x)\right]^{+}}{P_{1}(x)}, \frac{\left[P_{2}(x)\right]^{+}}{P_{2}(x)}, \frac{\left[P_{3}(x)\right]^{+}}{P_{3}(x)}\right)
$$

and

$$
\left[P_{0}\right]^{+}=\left\{\begin{array}{ll}
P_{0} & \text { if } P_{0}>0, \\
0 & \text { if } P_{0} \leq 0,
\end{array} \quad\left[P_{i}(x)\right]^{+}=\left\{\begin{array}{ll}
P_{i}(x) & \text { if } P_{i}(x)>0, \\
0 & \text { if } P_{i}(x) \leq 0,
\end{array} \quad i=1,2,3\right.\right.
$$

The boundary condition on $\mathbf{P} \in D(\mathbb{A})$ imply

$$
\begin{aligned}
& {\left[P_{1}(0)\right]^{+} \leq \lambda^{\prime}\left[P_{0}\right]^{+}+\int_{0}^{\infty} \eta(x)\left[P_{3}(x)\right]^{+} d x,} \\
& {\left[P_{2}(0)\right]^{+} \leq \lambda\left[P_{0}\right]^{+}} \\
& {\left[P_{3}(0)\right]^{+} \leq \lambda \int_{0}^{\infty}\left[P_{1}(x)\right]^{+} d x .}
\end{aligned}
$$

If we define $\mathcal{V}=\{x \in[0, \infty) \mid y(x)>0\}$ and $\mathcal{W}=\{x \in[0, \infty) \mid y(x) \leq 0\}$ then, replacing $y(x)$ in $\mathcal{V}, \mathcal{W}$ by $P_{1}(x), P_{2}(x), P_{3}(x)$ respectively, we have

$$
\int_{0}^{\infty} \frac{d P_{i}(x)}{d x} \frac{\left[P_{i}(x)\right]^{+}}{P_{1}(x)} d x
$$




$$
\begin{aligned}
& =\int_{V} \frac{d P_{i}(x)}{d x} \frac{\left[P_{i}(x)\right]^{+}}{P_{i}(x)} d x+\int_{W} \frac{d P_{i}(x)}{d x} \frac{\left[P_{i}(x)\right]^{+}}{P_{i}(x)} d x \\
& =\int_{V} \frac{d P_{i}(x)}{d x} \frac{\left[P_{i}(x)\right]^{+}}{P_{i}(x)} d x=\int_{V} \frac{d P_{i}(x)}{d x} d x \\
& =\int_{V} \frac{d\left[P_{i}(x)\right]^{+}}{d x} d x=-\left[P_{i}(0)\right]^{+}, \quad i=1,2,3 .
\end{aligned}
$$

By using the boundary condition on $\mathbf{P} \in D(\mathbb{A})$ and (A.21)-(A.23) for such $\xi$, we derive

$$
\begin{aligned}
\langle(\mathbb{A}+ & \mathbb{E}) \mathbf{P}, \xi\rangle \\
= & \left\{-\left(\lambda+\lambda^{\prime}\right) P_{0}+\int_{0}^{\infty} \varphi(x) P_{1}(x) d y+\int_{0}^{\infty} \eta(x) P_{2}(x) d x\right\} \frac{\left[P_{0}\right]^{+}}{P_{0}} \\
& +\int_{0}^{\infty}\left\{-\frac{d P_{1}(x)}{d x}-(\lambda+\varphi(x)) P_{1}(x)\right\} \frac{\left[P_{1}(x)\right]^{+}}{P_{1}(x)} d x \\
& +\int_{0}^{\infty}\left\{-\frac{d P_{2}(x)}{d x}-\eta(x) P_{2}(x)\right\} \frac{\left[P_{3}(x)\right]^{+}}{P_{3}(x)} d x \\
& +\int_{0}^{\infty}\left\{-\frac{d P_{2}(x)}{d x}-\eta(x) P_{2}(x)\right\} \frac{\left[P_{2}(x)\right]^{+}}{P_{2}(x)} d x \\
= & -\left(\lambda+\lambda^{\prime}\right)\left[P_{0}\right]^{+}+\left\{\int_{0}^{\infty} \varphi(x) P_{1}(x) d x+\int_{0}^{\infty} \eta(x) P_{2}(x) d x\right\} \frac{\left[P_{0,0}\right]^{+}}{P_{0,0}} \\
& +\left[P_{1}(0)\right]^{+}-\int_{0}^{\infty}(\lambda+\varphi(x))\left[P_{1}(x)\right]^{+} d x \\
& +\left[P_{2}(0)\right]^{+}-\int_{0}^{\infty} \eta(x)\left[P_{2}(x)\right]^{+} d x+\left[P_{3}(0)\right]^{+}-\int_{0}^{\infty} \eta(x)\left[P_{3}(x)\right]^{+} d x \\
\leq & -\left(\lambda+\lambda^{\prime}\right)\left[P_{0}\right]^{+}\left\{\int_{0}^{\infty} \varphi(x)\left[P_{1}(x)\right]^{+} d x+\int_{0}^{\infty} \eta(x)\left[P_{2}(x)\right]^{+} d x\right\} \frac{\left[P_{0,0}\right]^{+}}{P_{0,0}} \\
& +\lambda^{\prime}\left[P_{0}\right]^{+}+\int_{0}^{\infty} \eta(x)\left[P_{3}(x)\right]^{+} d x-\int_{0}^{\infty}(\lambda+\varphi(x))\left[P_{1}(x)\right]^{+} d x \\
& +\lambda\left[P_{0}\right]^{+}-\int_{0}^{\infty} \eta(x)\left[P_{2}(x)\right]^{+} d x+\lambda \int_{0}^{\infty}\left[P_{1}(x)\right]^{+} d x-\int_{0}^{\infty} \eta(x)\left[P_{3}(x)\right]^{+} d x \\
= & {\left.\left[\frac{\left[P_{0,0}\right]^{+}}{P_{0,0}}-1\right) \int_{0}^{\infty} \varphi(x)\left[P_{1}(x)\right]^{+} d x+\left(\frac{\left[P_{0,0}\right]^{+}}{P_{0,0}}-1\right) \int_{0}^{\infty} \eta(x) d x\left[P_{2}(x)\right]^{+}\right\} } \\
\leq & 0 .
\end{aligned}
$$

Expression (A.25) implies that $\mathbb{A}+\mathbb{E}$ is a dispersive operator.

From the above fourth steps and Fillips theorem (see Nagel [30]), we obtain that $\mathbb{A}+\mathbb{E}$ generates a positive contraction $C_{0}$-semigroup $\mathbb{T}(t)$.

Proof of Lemma 4.4 By using integration by parts and the boundary conditions on $P \in$ $D(\mathbb{A})$, we have, for $Q^{*} \in D\left(\mathbb{G}^{*}\right)$,

$$
\begin{aligned}
& \left\langle(\mathbb{A}+\mathbb{E}) \mathbf{P}, \mathbf{Q}^{*}\right\rangle \\
& \quad=\left[-\left(\lambda+\lambda^{\prime}\right) P_{0}+\int_{0}^{\infty} \varphi(x) P_{1}(x) d x+\int_{0}^{\infty} \eta(x) P_{2}(x) d x\right] Q_{0}^{*}
\end{aligned}
$$




$$
\begin{aligned}
& +\int_{0}^{\infty}\left[-\frac{d P_{1}(x)}{d x}-(\lambda+\varphi(x)) P_{1}(x)\right] Q_{1}^{*}(x) d x \\
& +\int_{0}^{\infty}\left[-\frac{d P_{2}(x)}{d x}-\eta(x) P_{2}(x)\right] Q_{2}^{*}(x) d x \\
& +\int_{0}^{\infty}\left[-\frac{d P_{3}(x)}{d x}-\eta(x) P_{3}(x)\right] Q_{3}^{*}(x) d x \\
=P_{0} & \left.-\left(\lambda+\lambda^{\prime}\right) Q_{0}^{*}\right] \\
& +\int_{0}^{\infty} P_{1}(x)\left[\varphi(x) Q_{0}^{*}\right] d x+\int_{0}^{\infty} P_{2}(x)\left[\eta(x) Q_{0}^{*}\right] d y \\
& +Q_{1}^{*}(0)\left[\lambda^{\prime} P_{0}+\int_{0}^{\infty} P_{3}(x) \eta(x) d x\right] \\
& +\int_{0}^{\infty} P_{1}(x)\left[\frac{d Q_{1}^{*}(x)}{d x}-(\lambda+\varphi(x)) Q_{1}^{*}(x)\right] d x \\
& +Q_{2}^{*}(0) \lambda P_{0}+\int_{0}^{\infty} P_{2}(x)\left[\frac{d Q_{2}^{*}(x)}{d x}-\eta(x) Q_{2}^{*}(x)\right] d x \\
& +Q_{3}^{*}(0) \int_{0}^{\infty} P_{1}(x) d x+\int_{0}^{\infty} P_{3}(x)\left[\frac{d Q_{3}^{*}(x)}{d x}-\eta(x) Q_{3}^{*}(x)\right] d x \\
= & P_{0}\left[-\left(\lambda+\lambda^{\prime}\right) Q_{0}^{*}+\lambda^{\prime} Q_{1}^{*}(0)+\lambda Q_{2}^{*}(0)\right] \\
& +\int_{0}^{\infty} P_{1}(x)\left[\frac{d Q_{1}^{*}(x)}{d x}-(\lambda+\varphi(x)) Q_{1}^{*}(x)+\varphi(x) Q_{0}^{*}+\lambda Q_{3}^{*}(0)\right] d x \\
& +\int_{0}^{\infty} P_{2}(x)\left[\frac{d Q_{2}^{*}(x)}{d x}-\eta(x) Q_{2}^{*}(x)+\eta(x) Q_{0}^{*}\right] d x \\
& +\int_{0}^{\infty} P_{3}(x)\left[\frac{d Q_{3}^{*}(x)}{d x}-\eta(x) Q_{3}^{*}(x)+\eta(x) Q_{1}^{*}(0)\right] d x \\
& \left(\mathbb{Q} \mathbf{Q}^{*}\right\rangle .
\end{aligned}
$$

From this calculation and the definition of the adjoint operator, we obtain the desired result.

\section{Acknowledgements}

The authors would like to thank the editor and referees for their valuable comments.

Funding

The author' research work was supported by the National Natural Science Foundation of China (No: 11801485).

\section{Abbreviations}

Not applicable.

\section{Availability of data and materials}

Not applicable.

\section{Competing interests}

The authors declare that there is no conflict of interest regarding the publication of this paper.

\section{Authors' contributions}

The authors equally contributed in preparing this manuscript. All authors read and approved the final manuscript.

\section{Publisher's Note}

Springer Nature remains neutral with regard to jurisdictional claims in published maps and institutional affiliations.

Received: 14 December 2019 Accepted: 20 March 2020 Published online: 06 April 2020 


\section{References}

1. Cox, D.R.: The analysis of non-Markovian stochastic processes by the inclusion of supplementary variables. Math. Proc. Camb. Philos. Soc. 51, 433-441 (1955)

2. Gaver, D.P.: Time to failure and availability of parallel redundant systems with repair. IEEE Trans. Reliab. R-12, 30-38 (1963)

3. Linton, D.G.: Some advancements in the analysis of two-unit parallel redundant systems. Microelectron. Reliab. 15(1), 39-46 (1976)

4. Goel, L.R., Gupta, R., Singh, S.K.: Availability analysis of a two-unit (dissimilar) parallel system with inspection and bivariate exponential life times. Microelectron. Reliab. 25(1), 77-80 (1985)

5. Gupta, P.P., Sharma, R.K.: Cost analysis of a three-state standby redundant electronic equipment. Microelectron. Reliab. 25(6), 1029-1033 (1985)

6. Shi, D.H., Li, W.: Availability analysis of a two-unit series system with shut-off rule and "first-fail, first-repaired". Acta Math. Appl. Sin. Engl. Ser. 9(1), 88-91 (1993)

7. Chung, W.K.: Reliability of imperfect switching of cold stanby systems with multiple non-critical and critical errors Microelectron. Reliab. 35(12), 1479-1482 (1995)

8. Zhang, Y.L., Wu, S.M.: Reliability analysis for a $k / n(f)$ system with repairable repair-equipment. Appl. Math. Model. 33(7), 3052-3067 (2009)

9. Dhillon, B.S., Shen, C.: Reliability and availability analysis of a robot-safety system. J. Qual. Maint. Eng. 17(2), 203-232 (2011)

10. Ram, M., Singh, S.B., Singh, V.V.: Stochastic analysis of a standby system with waiting repair strategy. IEEE Trans. Syst. Man Cybern. Syst. 43(3), 698-707 (2013)

11. Zhang, N., Wang, J.T.: Reliability analysis of unrepairable systems with k-out-of-m:g subsystems subject to suspended animation. Commun. Stat., Simul. Comput. 43(8), 1900-1912 (2014)

12. Ke, J.C., Liu, T.H., Yang, D.Y.: Machine repairing systems with standby switching failure. Comput. Ind. Eng. 99, 223-228 (2016)

13. Gupur, G.: Well-posedness of the system consisting of two repairable units. Acta Anal. Funct. Appl. 3(2), 188-192 (2001) (in Chinese)

14. Gupur, G., Li, X.Z.: Semigroup method for a mathematical model in reliability analysis. J. Syst. Sci. Syst. Eng. 10(2) 137-147 (2001)

15. Gupur, G., Wong, M.W.: On a dynamic system for a reliability model. J. Pseudo-Differ. Oper. Appl. 2(3), 509-542 (2011)

16. Gupur, G.: Point spectrum of the operator corresponding to a reliability model and application. J. Pseudo-Differ. Oper. Appl. 7(3), 411-429 (2016)

17. Gupur, G.: Well-posedness of the model describing a repairable, standby, human \& machine system. J. Syst. Sci. Complex. 16(4), 483-493 (2003)

18. Gupur, G.: Asymptotic property of the solution of a repairable, standby, human and machine system. Int. J. Pure Appl. Math. 28(1), 35-54 (2006)

19. Aili, M., Gupur, G.: Further result on a repairable, standby, human and machine system. Int. J. Pure Appl. Math. 101(4), 571-594 (2015)

20. Ehmet, R., Gupur, G.: Further research of k-out-of-N:G redundant system with repair and multiple critical and non-critical errors. Int. J. Pure Appl. Math. 51(3), 325-343 (2009)

21. Ablet, E., Gupur, G.: Expression and application of the project operator appearing during research of k-out-of-N:G redundant system with repair and multiple critical and non-critical errors. Acta Anal. Funct. Appl. 12(4), 310-321 (2010)

22. Gupur, G.: Functional Analysis Methods for Reliability Models. Springer, Basel (2011)

23. Guo, L.N., Xu, H.B., Gao, C., Zhu, G.T.: Stability analysis of a new kind n-unit series repairable system. Appl. Math. Model. 35(1), 202-217 (2011)

24. Wang, H.X., Xu, G.Q.: A cold system with two different components and a single vacation of the repairman. Appl. Math. Comput. 219(5), 2634-2657 (2012)

25. Haji, A., Radl, A.: A semigroup approach to the Gnedenko system with single vacation of a repairman. Semigroup Forum 86(1), 41-58 (2013)

26. Hu, W.W.: Differentiability and compactness of the $c_{0}$-semigroup generated by the reparable system with finite repair time. J. Math. Anal. Appl. 433(2), 1614-1625 (2016)

27. Govil, A.K.: Priority effect on pointwise availability of the system. RAIRO Oper. Res. 6(V1), 47-56 (1972)

28. Gupur, G.: On asymptotic behavior of the time-dependent solution of a reliability model. Int Front. Sci. Lett. 2, 1-11 (2014)

29. Fattorini, H.O.: The Cauchy Problem. Cambridge University Press, Cambridge (1984)

30. Nagel, R.: One-Parameter Semigroups of Positive Operators (LNM 1184). Springer, Berlin (1986)

31. Engel, K.J., Nagel, R.: One-Parameter Semigroups for Linear Evolution Equations. Springer, New York (2000) 